Research Article

Marat V. Markin*

\title{
On the Gevrey ultradifferentiability of weak solutions of an abstract evolution equation with a scalar type spectral operator on the real axis
}

https://doi.org/10.1515/math-2020-0128

received September 16, 2020; accepted December 21, 2020

\begin{abstract}
Given the abstract evolution equation $y^{\prime}(t)=A y(t), t \in \mathbb{R}$, with a scalar type spectral operator $A$ in a complex Banach space, we find conditions on $A$, formulated exclusively in terms of the location of its spectrum in the complex plane, necessary and sufficient for all weak solutions of the equation, which a priori need not be strongly differentiable, to be strongly Gevrey ultradifferentiable of order $\beta \geq 1$, in particular analytic or entire, on $\mathbb{R}$. We also reveal certain inherent smoothness improvement effects and show that if all weak solutions of the equation are Gevrey ultradifferentiable of orders less than one, then the operator $A$ is necessarily bounded. The important particular case of the equation with a normal operator $A$ in a complex Hilbert space follows immediately.
\end{abstract}

Keywords: weak solution, scalar type spectral operator, Gevrey classes

MSC 2020: 34G10, 47B40, 30D60, 30D15, 47B15, 47D06, 47D60

\section{Introduction}

We find conditions on a scalar type spectral operator $A$ in a complex Banach space, formulated exclusively in terms of the location of its spectrum in the complex plane, necessary and sufficient for all weak solutions of the evolution equation

$$
y^{\prime}(t)=A y(t), \quad t \in \mathbb{R},
$$

which a priori need not be strongly differentiable, to be strongly Gevrey ultradifferentiable of order $\beta \geq 1$, in particular analytic or entire, on $\mathbb{R}$. We also reveal certain inherent smoothness improvement effects and show that if all weak solutions of the equation are Gevrey ultradifferentiable of orders less than one, then the operator $A$ is necessarily bounded.

The important particular case of the equation with a normal operator $A$ in a complex Hilbert space follows immediately.

We proceed along the path of developing the results of paper [1] on the strong differentiability of the weak solutions of equation (1.1) on $\mathbb{R}$ and of papers [2-4], where similar consideration is given to the Gevrey ultradifferentiability of the weak solutions of the evolution equation

$$
y^{\prime}(t)=A y(t), \quad t \geq 0
$$

on $[0, \infty)$ and $(0, \infty)$.

\footnotetext{
* Corresponding author: Marat V. Markin, Department of Mathematics, California State University, Fresno, 5245 N. Backer Avenue, M/S PB 108, Fresno, CA 93740-8001, USA, e-mail: mmarkin@csufresno.edu
} 


\section{Preliminaries}

Here, we briefly outline certain facts essential for the subsequent discourse (for more details, see, e.g., $[1-3,5])$.

\subsection{Weak solutions}

Definition 2.1. (Weak solution)

Let $A$ be a densely defined closed linear operator in a Banach space $(X,\|\cdot\|)$ and $I$ be an interval of the real axis $\mathbb{R}$. A strongly continuous vector function $y: I \rightarrow X$ is called a weak solution of the evolution equation

$$
y^{\prime}(t)=A y(t), \quad t \in I,
$$

if, for any $g^{\star} \in D\left(A^{\star}\right)$,

$$
\frac{\mathrm{d}}{\mathrm{d} t}\left\langle y(t), g^{\star}\right\rangle=\left\langle y(t), A^{\star} g^{\star}\right\rangle, \quad t \in I,
$$

where $D(\cdot)$ is the domain of an operator, $A^{\star}$ is the operator adjoint to $A$, and $\langle\cdot, \cdot\rangle$ is the pairing between the space $X$ and its dual $X^{\star}$ (cf. [6]).

\section{Remarks 2.1.}

- Due to the closedness of $A$, a weak solution of equation (2.3) can be equivalently defined to be a strongly continuous vector function $y: I \mapsto X$ such that, for all $t \in I$,

$$
\int_{t_{0}}^{t} y(s) \mathrm{d} s \in D(A) \text { and } y(t)=y\left(t_{0}\right)+A \int_{t_{0}}^{t} y(s) \mathrm{d} s,
$$

where $t_{0}$ is an arbitrary fixed point of the interval $I$, and is also called a mild solution (cf. [7, Ch. II, Definition 6.3], see also [5, Preliminaries]).

- Such a notion of weak solution, which need not be differentiable in the strong sense, generalizes that of classical one, strongly differentiable on $I$ and satisfying the equation in the traditional plug-in sense, the classical solutions being precisely the weak ones strongly differentiable on $I$.

- As is easily seen $y: \mathbb{R} \rightarrow X$ is a weak solution of equation (1.1) iff

(i)

$$
y_{+}(t):=y(t), \quad t \geq 0,
$$

is a weak solution of equation (1.2) and

$$
y_{-}(t):=y(-t), \quad t \geq 0,
$$

is a weak solution of the equation

$$
y^{\prime}(t)=-A y(t), \quad t \geq 0,
$$

or

(ii)

$$
y_{-}(t):=y(-t), \quad t \in \mathbb{R},
$$

is a weak solution of the equation

$$
y^{\prime}(t)=-A y(t), \quad t \in \mathbb{R} .
$$


- When a closed densely defined linear operator $A$ in a complex Banach space $X$ generates a strongly continuous group $\{T(t)\}_{t \in \mathbb{R}}$ of bounded linear operators (see, e.g., [7,8]), i.e., the associated abstract Cauchy problem $(A C P)$

$$
\left\{\begin{array}{l}
y^{\prime}(t)=A y(t), \quad t \in \mathbb{R} \\
y(0)=f
\end{array}\right.
$$

is well posed (cf. [7, Ch. II, Definition 6.8]), the weak solutions of equation (1.1) are the orbits

$$
y(t)=T(t) f, \quad t \in \mathbb{R},
$$

with $f \in X$ (cf. [7, Ch. II, Proposition 6.4], see also [6, Theorem]), whereas the classical ones are those with $f \in D(A)$ (see, e.g., [7, Ch. II, Proposition 6.3]).

\subsection{Scalar type spectral operators}

Henceforth, unless specified otherwise, $A$ is a scalar type spectral operator in a complex Banach space $(X,\|\cdot\|)$ with strongly $\sigma$-additive spectral measure (the resolution of the identity) $E_{A}(\cdot)$ assigning to Borel sets of the complex plane $\mathbb{C}$ bounded projection operators on $X$ and having the operator's spectrum $\sigma(A)$ as its support [9-11].

Observe that, in a complex Hilbert space, the scalar type spectral operators are precisely all those that are similar to the normal ones [12-14].

Associated with a scalar type spectral operator $A$ is the Borel operational calculus assigning to each complex-valued Borel measurable function $F: \sigma(A) \rightarrow \mathbb{C}$ a scalar type spectral operator

$$
F(A):=\int_{\sigma(A)} F(\lambda) \mathrm{d} E_{A}(\lambda)
$$

[11]. In particular,

$$
A^{n}=\int_{\sigma(A)} \lambda^{n} \mathrm{~d} E_{A}(\lambda), \quad n \in \mathbb{Z}_{+}, \quad \text { and } e^{t A}:=\int_{\sigma(A)} e^{t \lambda} \mathrm{d} E_{A}(\lambda), \quad t \in \mathbb{R},
$$

$\left(\mathbb{Z}_{+}:=\{0,1,2, \ldots\}\right.$ is the set of nonnegative integers, $A^{0}:=I, I$ is the identity operator on $\left.X\right)$.

Provided

$$
\sigma(A) \subseteq\{\lambda \in \mathbb{C} \mid \operatorname{Re} \lambda \leq \omega\}
$$

with some $\omega \in \mathbb{R}$, the collection of exponentials $\left\{e^{t A}\right\}_{t \geq 0}$ is the $C_{0}$-semigroup generated by $A$ [15, Proposition 3.1] (cf. also [16,17]), and hence, if

$$
\sigma(A) \subseteq\{\lambda \in \mathbb{C} \mid-\omega \leq \operatorname{Re} \lambda \leq \omega\}
$$

with some $\omega \geq 0$, the collection of exponentials $\left\{e^{t A}\right\}_{t \in \mathbb{R}}$ is the strongly continuous group of bounded linear operators generated by $A$.

Being strongly $\sigma$-additive, the spectral measure is bounded, i.e., there exists an $M \geq 1$ such that, for any Borel set $\delta \subseteq \mathbb{C}$,

$$
\left\|E_{A}(\delta)\right\| \leq M
$$

$[11,18]$.

Remark 2.2. The notation $\|\cdot\|$ is used here to designate the norm on the space $L(X)$ of all bounded linear operators on $X$. Henceforth, we adhere to this rather conventional economy of symbols adopting the same notation also for the norm on the dual space $X^{\star}$. 
For arbitrary Borel measurable function $F: \mathbb{C} \rightarrow \mathbb{C}, f \in D(F(A)), g^{\star} \in X^{\star}$, and Borel set $\delta \subseteq \mathbb{C}$,

$$
\int_{\delta}|F(\lambda)| \mathrm{d} v\left(f, g^{\star}, \lambda\right) \leq 4 M\left\|E_{A}(\delta) F(A) f\right\|\left\|g^{\star}\right\|,
$$

where $v\left(f, g^{\star}, \cdot\right)$ is the total variation measure of the complex-valued Borel measure $\left\langle E_{A}(\cdot) f, g^{\star}\right\rangle$, for which

$$
v\left(f, g^{\star}, \mathbb{C}\right)=v\left(f, g^{\star}, \sigma(A)\right) \leq 4 M\|f\|\left\|g^{\star}\right\|,
$$

where $M \geq 1$ in (2.9) and (2.10) is from (2.8) (see, e.g., [19,20]).

In particular, for $\delta=\sigma(A), E_{A}(\delta)=I$ (see, e.g., [11]), (2.9) turns into

$$
\int_{\sigma(A)}|F(\lambda)| \mathrm{d} v\left(f, g^{\star}, \lambda\right) \leq 4 M\|F(A) f\|\left\|g^{\star}\right\| .
$$

Furthermore (see, e.g., [1,2]), for arbitrary Borel measurable function $F: \mathbb{C} \rightarrow[0, \infty$ ), Borel set $\delta \subseteq \mathbb{C}$, sequence $\left\{\Delta_{n}\right\}_{n=1}^{\infty}$ of pairwise disjoint Borel sets in $\mathbb{C}, f \in X$, and $g^{\star} \in X^{\star}$,

$$
\int_{\delta} F(\lambda) \mathrm{d} v\left(E_{A}\left(\cup_{n=1}^{\infty} \Delta_{n}\right) f, g^{\star}, \lambda\right)=\sum_{n=1}^{\infty} \int_{\delta \cap \Delta_{n}} F(\lambda) \mathrm{d} v\left(E_{A}\left(\Delta_{n}\right) f, g^{\star}, \lambda\right) .
$$

Remark 2.3. Subsequently, the frequently used term "spectral measure" is abbreviated to s.m.

The following statement characterizing the domains of Borel measurable functions of a scalar type spectral operator in terms of Borel measures is fundamental for our discourse.

Proposition 2.1. [21, Proposition 3.1]

Let $A$ be a scalar type spectral operator in a complex Banach space $(X,\|\cdot\|)$ with spectral measure $E_{A}(\cdot)$ and $F: \sigma(A) \rightarrow \mathbb{C}$ be a Borel measurable function. Then $f \in D(F(A))$ iff

(i) for each $g^{\star} \in X^{\star}, \int_{\sigma(A)}|F(\lambda)| \mathrm{d} v\left(f, g^{\star}, \lambda\right)<\infty$ and

(ii) $\sup _{\left\{g^{\star} \in X^{\star} \mid\left\|g^{\star}\right\|=1\right\}} \int_{\{\lambda \in \sigma(A) \| F(\lambda) \mid>n\}}|F(\lambda)| \mathrm{d} v\left(f, g^{\star}, \lambda\right) \rightarrow 0, \quad n \rightarrow \infty$, where $v\left(f, g^{\star}, \cdot\right)$ is the total variation measure of $\left\langle E_{A}(\cdot) f, g^{\star}\right\rangle$.

The succeeding key theorem provides a description of the weak solutions of equation (1.1) with a scalar type spectral operator $A$ in a complex Banach space.

Theorem 2.1. [1, Theorem 7]

Let $A$ be a scalar type spectral operator in a complex Banach space $(X,\|\cdot\|)$. A vector function $y: \mathbb{R} \rightarrow X$ is a weak solution of equation (1.1) iff there exists an $f \in \bigcap_{t \in \mathbb{R}} D\left(e^{t A}\right)$ such that

$$
y(t)=e^{t A} f, \quad t \in \mathbb{R},
$$

the operator exponentials understood in the sense of the Borel operational calculus (see (2.7)).

We also need the following characterization of a particular weak solution of equation (1.1) with a scalar type spectral operator $A$ in a complex Banach space being strongly infinite differentiable on a subinterval $I$ of $\mathbb{R}$.

Proposition 2.2. [1, Corollary 11]

Let $A$ be a scalar type spectral operator in a complex Banach space $(X,\|\cdot\|)$ and I be interval of the real axis $\mathbb{R}$. A weak solution $y(\cdot)$ of equation (1.1) is strongly infinite differentiable on $I\left(y(\cdot) \in C^{\infty}(I, X)\right)$ iff, for each $t \in I$,

$$
y(t) \in C^{\infty}(A):=\bigcap_{n=0}^{\infty} D\left(A^{n}\right),
$$

in which case

$$
y^{(n)}(t)=A^{n} y(t), \quad n \in \mathbb{N}, t \in I .
$$




\subsection{Gevrey classes of functions}

Definition 2.2. (Gevrey classes of functions)

Let $(X,\|\cdot\|)$ be a (real or complex) Banach space, $C^{\infty}(I, X)$ be the space of all $X$-valued functions strongly infinite differentiable on an interval $I$ of the real axis $\mathbb{R}$, and $0 \leq \beta<\infty$.

The following subspaces

$$
\mathscr{E}^{\{\beta\}}(I, X):=\left\{g(\cdot) \in C^{\infty}(I, X) \mid \forall[a, b] \subseteq I \exists \alpha>0 \exists c>0: \max _{a \leq t \leq b}\left\|g^{(n)}(t)\right\| \leq c \alpha^{n}(n !)^{\beta}, n \in \mathbb{Z}_{+}\right\}
$$

and

$$
\mathscr{E}^{(\beta)}(I, X):=\left\{g(\cdot) \in C^{\infty}(I, X) \mid \forall[a, b] \subseteq I \forall \alpha>0 \exists c>0: \max _{a \leq t \leq b}\left\|g^{(n)}(t)\right\| \leq c \alpha^{n}(n !)^{\beta}, n \in \mathbb{Z}_{+}\right\}
$$

of $C^{\infty}(I, X)$ are called the $\beta$ th-order Gevrey classes of strongly ultradifferentiable vector functions on $I$ of Roumieu and Beurling type, respectively (see, e.g., [22-25]).

\section{Remarks 2.4.}

- In view of Stirling's formula, the sequence $\left\{(n !)^{\beta}\right\}_{n=0}^{\infty}$ can be replaced with $\left\{n^{\beta m_{n=0}^{\infty}}\right.$.

- For $0 \leq \beta<\beta^{\prime}<\infty$, the inclusions

$$
\mathscr{E}^{(\beta)}(I, X) \subseteq \mathscr{E}^{\{\beta\}}(I, X) \subseteq \mathscr{E}^{\left(\beta^{\prime}\right)}(I, X) \subseteq \mathscr{E}^{\left\{\beta^{\prime}\right\}}(I, X) \subseteq C^{\infty}(I, X)
$$

hold.

- For $1<\beta<\infty$, the Gevrey classes $\mathscr{E}^{(\beta)}(I, X)$ and $\mathscr{E}^{\{\beta\}}(I, X)$ are non-quasianalytic (see, e.g., [24]).

- The first-order Roumieu-type Gevrey class $\mathscr{E}^{\{1\}}(I, X)$ consists of all analytic on $I$, i.e., analytically continuable into complex neighborhoods of $I$, vector functions and the first-order Beurling-type Gevrey class $\mathscr{E}^{(1)}(I, X)$ consists of all entire, i.e., allowing entire continuations, vector functions [26].

- For $0 \leq \beta<1$, the Roumieu-type Gevrey class $\mathscr{E}^{\{\beta\}}(I, X)$ (the Beurling-type Gevrey class $\left.\mathscr{E}^{(\beta)}(I, X)\right)$ consists of all functions $g(\cdot) \in \mathscr{E}^{(1)}(I, X)$ such that, for some (any) $\gamma>0$, there exists an $M>0$, for which

$$
\|g(z)\| \leq M e^{\gamma|z|^{1 /(1-\beta)}}, \quad z \in \mathbb{C},
$$

[27]. In particular, the Gevrey classes $\mathscr{E}^{\{0\}}(I, X)$ and $\mathscr{E}^{(0)}(I, X)$ are the classes of entire vector functions of exponential and minimal exponential type, respectively (see, e.g., [28]).

\subsection{Gevrey classes of vectors}

Definition 2.3. (Gevrey classes of vectors)

Let $A$ be a densely defined closed linear operator in a (real or complex) Banach space $(X,\|\cdot\|)$ and $0 \leq \beta<\infty$.

The following subspaces

$$
\mathscr{E}^{\{\beta\}}(A):=\left\{f \in C^{\infty}(A) \mid \exists \alpha>0 \exists c>0:\left\|A^{n} f\right\| \leq c \alpha^{n}(n !)^{\beta}, n \in \mathbb{Z}_{+}\right\}
$$

and

$$
\mathscr{E}^{\mathscr{C}(\beta)}(A):=\left\{f \in C^{\infty}(A) \mid \forall \alpha>0 \exists c>0:\left\|A^{n} f\right\| \leq c \alpha^{n}(n !)^{\beta}, n \in \mathbb{Z}_{+}\right\}
$$

of $C^{\infty}(A)$ are called the $\beta$ th-order Gevrey classes of ultradifferentiable vectors of $A$ of Roumieu and Beurling type, respectively (see, e.g., [29-31]). 


\section{Remarks 2.5.}

- In view of Stirling's formula, the sequence $\left\{(n !)^{\beta}\right\}_{n=0}^{\infty}$ can be replaced with $\left\{n^{\beta n}\right\}_{n=0}^{\infty}$.

- For $0 \leq \beta<\beta^{\prime}<\infty$, the inclusions

$$
\mathscr{E}^{(\beta)}(A) \subseteq \mathscr{E}^{\{\beta\}}(A) \subseteq \mathscr{E}^{\left(\beta^{\prime}\right)}(A) \subseteq \mathscr{E}^{\left\{\beta^{\prime}\right\}}(A) \subseteq C^{\infty}(A)
$$

hold.

- In particular, $\mathscr{E}^{\{1\}}(A)$ and $\mathscr{E}^{(1)}(A)$ are the classes of analytic and entire vectors of $A$, respectively [32,33] and $\mathscr{E}^{\{0\}}(A)$ and $\mathscr{E}^{(0)}(A)$ are the classes of entire vectors of $A$ of exponential and minimal exponential type, respectively (see, e.g., [31,34]).

- As is readily seen, in view of the closedness of $A$, the first-order Beurling-type Gevrey class $\mathscr{E}^{(1)}(A)$ forms the subspace of the initial values $f \in X$ generating the (classical) solutions of (1.1), which are entire vector functions represented by the power series

$$
\sum_{n=0}^{\infty} \frac{t^{n}}{n !} A^{n} f, \quad t \in \mathbb{R},
$$

the classes $\mathscr{E}^{\{\beta\}}(A)$ and $\mathscr{E}^{(\beta)}(A)$ with $0 \leq \beta<1$ being the subspaces of such initial values for which the solutions satisfy growth estimate (2.14) with some (any) $y>0$ and some $M=M(\gamma)>0$, respectively (cf. [28]).

As is shown in [29] (see also [30,31]), for a normal operator $A$ in a complex Hilbert space and any $0<\beta<\infty$,

$$
\mathscr{E}^{\{(\beta\}}(A)=\bigcup_{t>0} D\left(e^{t|A|^{1 / \beta}}\right) \text { and } \mathscr{E}^{(\beta)}(A)=\bigcap_{t>0} D\left(e^{t|A|^{1 / \beta}}\right)
$$

the operator exponentials $e^{t|A|^{1 / \beta}}, t>0$, understood in the sense of the Borel operational calculus (see, e.g., $[12,13])$.

In $[20,35]$, descriptions (2.15) are extended to scalar type spectral operators in a complex Banach space. In [35], similar nature descriptions of the classes $\mathscr{E}^{\{0\}}(A)$ and $\mathscr{E}^{(0)}(A)(\beta=0)$, known for a normal operator $A$ in a complex Hilbert space (see, e.g., [31]), are also generalized to scalar type spectral operators in a complex Banach space. In particular [35, Theorem 5.1],

$$
\mathscr{E}^{\{0\}}(A)=\bigcup_{\alpha>0} E_{A}\left(\Delta_{\alpha}\right) X
$$

where

$$
\Delta_{\alpha}:=\{\lambda \in \mathbb{C}|| \lambda \mid \leq \alpha\}, \quad \alpha>0
$$

\subsection{Gevrey ultradifferentiability of a particular weak solution of (1.2)}

We also need the following characterization of a particular weak solution of equation (1.1) with a scalar type spectral operator $A$ in a complex Banach space being strongly Gevrey ultradifferentiable on a subinterval $I$ of $[0, \infty)$.

Proposition 2.3. [2, Proposition 3.1]

Let $A$ be a scalar type spectral operator in a complex Banach space $(X,\|\cdot\|), 0 \leq \beta<\infty$, and I be a subinterval of $[0, \infty)$. The restriction of a weak solution $y(\cdot)$ of equation (1.2) to I belongs to the Gevrey class $\mathscr{E}^{\{\beta\}}(I, X)$ $\left(\mathscr{E}^{(\beta)}(I, X)\right)$ iff, for each $t \in I$,

$$
y(t) \in \mathscr{E}^{\{\beta\}}(A)\left(\mathscr{E}^{(\beta)}(A), \text { respectively }\right)
$$

in which case

$$
y^{(n)}(t)=A^{n} y(t), \quad n \in \mathbb{N}, t \in I
$$




\section{Gevrey ultradifferentiability of a particular weak solution}

Proposition 3.1. (Gevrey ultradifferentiability of a particular weak solution)

Let $A$ be a scalar type spectral operator in a complex Banach space $(X,\|\cdot\|), 0 \leq \beta<\infty$, and I be an interval of the real axis $\mathbb{R}$. The restriction of a weak solution $y(\cdot)$ of equation (1.1) to I belongs to the Gevrey class $\mathscr{E}^{\{\beta\}}(I, X)\left(\mathscr{E}^{(\beta)}(I, X)\right)$ iff, for each $t \in I$,

$$
y(t) \in \mathscr{E}^{\{\beta\}}(A)\left(\mathscr{E}^{\mathscr{C}(\beta)}(A), \text { respectively }\right),
$$

in which case

$$
y^{(n)}(t)=A^{n} y(t), \quad n \in \mathbb{N}, t \in I
$$

Proof. As is noted in Remarks 2.1, $y: \mathbb{R} \rightarrow X$ is a weak solution of (1.1) iff

$$
y_{+}(t):=y(t), t \geq 0,
$$

is a weak solution of equation (1.2) and

$$
y_{-}(t):=y(-t), t \geq 0,
$$

is a weak solution of equation (2.4).

The statement immediately follows from Proposition 2.3 applied to

$$
y_{+}(t):=y(t), t \geq 0, \quad \text { and } \quad y_{-}(t):=y(-t), t \geq 0,
$$

for an arbitrary weak solution $y(\cdot)$ of equation (1.1) in view of

$$
\mathscr{E}^{\{\beta\}}(-A)=\mathscr{E}^{\lfloor\beta\}}(A) \quad \text { and } \quad \mathscr{E}^{(\beta)}(-A)=\mathscr{E}^{(\beta)}(A) .
$$

\section{Gevrey ultradifferentiability of order $\beta \geq 1$}

Theorem 4.1. (Gevrey ultradifferentiability of order $\beta \geq 1$ )

Let $A$ be a scalar type spectral operator in a complex Banach space $(X,\|\cdot\|)$ with spectral measure $E_{A}(\cdot)$ and $1 \leq \beta<\infty$. Then the following statements are equivalent.

(i) Every weak solution of equation (1.1) belongs to the $\beta$ th-order Beurling-type Gevrey class $\mathscr{E}^{(\beta)}(\mathbb{R}, X)$.

(ii) Every weak solution of equation (1.1) belongs to the $\beta$ th-order Roumieu-type Gevrey class $\mathscr{E}^{\{\beta\}}(\mathbb{R}, X)$.

(iii) There exist $b_{+}>0$ and $b_{-}>0$ such that the set $\sigma(A) \backslash \mathscr{P}_{b_{-}, b_{+}}^{\beta}$, where

$$
\mathscr{P}_{b_{-}, b_{+}}^{\beta}:=\left\{\left.\lambda \in \mathbb{C}\left|\operatorname{Re} \lambda \leq-b_{-}\right| \operatorname{Im} \lambda\right|^{1 / \beta} \text { or } \operatorname{Re} \lambda \geq b_{+}|\operatorname{Im} \lambda|^{1 / \beta}\right\},
$$

is bounded (see Figure 1).

Proof. We are to prove the closed chain of implications

$$
\text { (i) } \Rightarrow \text { (ii) } \Rightarrow \text { (iii) } \Rightarrow \text { (i), }
$$

the implication (i) $\Rightarrow$ (ii) following immediately from the inclusion

$$
\mathscr{E}^{(\beta)}(\mathbb{R}, X) \subseteq \mathscr{E}^{\{\beta\}}(\mathbb{R}, X)
$$

(see Remarks 2.5).

To prove the implication (iii) $\Rightarrow$ (i), suppose that there exist $b_{+}>0$ and $b_{-}>0$ such that the $\operatorname{set} \sigma(A) \backslash \mathscr{P}_{b_{-}, b_{+}}^{\beta}$ is bounded and let $y(\cdot)$ be an arbitrary weak solution of equation (1.1). 


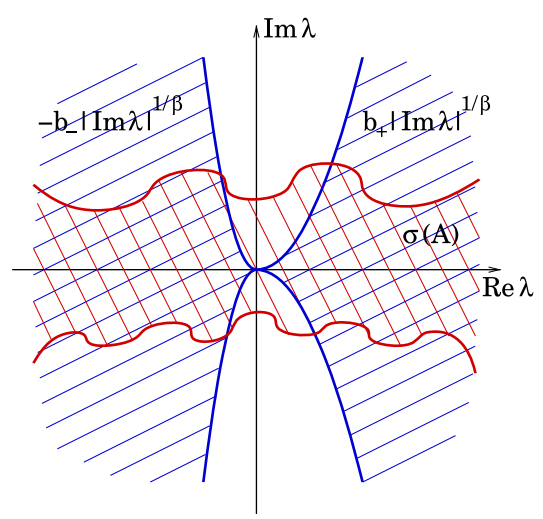

Figure 1: Gevrey ultradifferentiability of order $1 \leq \beta<\infty$.

By Theorem 2.1,

$$
y(t)=e^{t A} f, \quad t \in \mathbb{R}, \text { with some } f \in \bigcap_{t \in \mathbb{R}} D\left(e^{t A}\right) .
$$

Our purpose is to show that $y(\cdot) \in \mathscr{E}^{(\beta)}(\mathbb{R}, X)$, which, by Proposition 3.1 and (2.15), is accomplished by showing that, for each $t \in \mathbb{R}$,

$$
y(t) \in \mathscr{E}^{\mathscr{E}(\beta)}(A)=\bigcap_{s>0} D\left(e^{s|A|^{1 / \beta}}\right)
$$

Let us proceed by proving that, for any $t \in \mathbb{R}$ and $s>0$,

$$
y(t) \in D\left(e^{s|A|^{1 / \beta}}\right)
$$

via Proposition 2.1.

For any $s>0, t \in \mathbb{R}$ and an arbitrary $g^{\star} \in X^{\star}$,

$$
\begin{aligned}
\int_{\sigma(A)} e^{S|\lambda|^{1 / \beta}} e^{t \operatorname{Re} \lambda} \mathrm{d} v\left(f, g^{\star}, \lambda\right)= & \int_{\sigma(A) \backslash \mathscr{P}_{b_{-}, b_{+}}^{\beta}} e^{s|\lambda|^{1 / \beta}} e^{t \operatorname{Re} \lambda} \mathrm{d} v\left(f, g^{\star}, \lambda\right) \\
& +\int_{\left\{\lambda \in \sigma(A) \cap \mathscr{P}_{b_{-}, b_{+}}^{\beta} \mid-1<\operatorname{Re} \lambda<1\right\}} e^{s|\lambda|^{1 / \beta}} e^{t \operatorname{Re} \lambda} \mathrm{d} v\left(f, g^{\star}, \lambda\right) \\
& +\int_{\left\{\lambda \in \sigma(A) \cap \mathscr{P}_{b_{-}, b_{+}}^{\beta} \mid \operatorname{Re} \lambda \geq 1\right\}} e^{s|\lambda|^{1 / \beta}} e^{t \operatorname{Re} \lambda} \mathrm{d} v\left(f, g^{\star}, \lambda\right) \\
& +\int_{\left\{\lambda \in \sigma(A) \cap \mathscr{P}_{b_{-}, b_{+}}^{\beta} \mid \operatorname{Re} \lambda \leq-1\right\}} e^{s|\lambda|^{1 / \beta}} e^{t \operatorname{Re} \lambda} \mathrm{d} v\left(f, g^{\star}, \lambda\right)<\infty .
\end{aligned}
$$

Indeed,

$$
\int_{\sigma(A) \backslash \mathscr{P}_{b_{-}, b_{+}}^{\beta}} e^{S|\lambda|^{1 / \beta}} e^{t \operatorname{Re} \lambda} \mathrm{d} v\left(f, g^{\star}, \lambda\right)<\infty
$$

and

$$
\int_{\left\{\lambda \in \sigma(A) \cap \mathscr{P}_{b_{-}, b_{+}}^{\beta} \mid-1<\operatorname{Re} \lambda<1\right\}} e^{s|\lambda|^{1 / \beta}} e^{t \operatorname{Re} \lambda} \mathrm{d} v\left(f, g^{\star}, \lambda\right)<\infty
$$

due to the boundedness of the sets

$$
\sigma(A) \backslash \mathscr{P}_{b_{-}, b_{+}}^{\beta} \text { and } \quad\left\{\lambda \in \sigma(A) \cap \mathscr{P}_{b_{-}, b_{+}}^{\beta} \mid-1<\operatorname{Re} \lambda<1\right\},
$$


the continuity of the integrated function on $\mathbb{C}$, and the finiteness of the measure $v\left(f, g^{\star}, \cdot\right)$.

Further, for any $s>0, t \in \mathbb{R}$ and an arbitrary $g^{\star} \in X^{\star}$,

$$
\begin{aligned}
& \int_{\left\{\lambda \in \sigma(A) \cap \mathscr{P}_{b, b_{+}}^{\beta} \mid \operatorname{Re} \lambda \geq 1\right\}} e^{S|\lambda|^{1 / \beta}} e^{t \operatorname{Re} \lambda} \mathrm{d} v\left(f, g^{\star}, \lambda\right) \\
& \leq \int_{\left\{\lambda \in \sigma(A) \cap \mathscr{P}_{b_{-,}, b_{+}} \mid \operatorname{Re} \lambda \geq 1\right\}} e^{S[|\operatorname{Re} \lambda|+\mid \operatorname{Im} \lambda]^{1 / \beta}} e^{t \operatorname{Re} \lambda} \mathrm{d} v\left(f, g^{\star}, \lambda\right) \\
& \text { since, for } \lambda \in \sigma(A) \cap \mathscr{P}_{b_{,}, b_{+}}^{\beta} \text { with } \operatorname{Re} \lambda \geq 1, b_{+}^{-\beta} \operatorname{Re} \lambda^{\beta} \geq|\operatorname{Im} \lambda| \text {; } \\
& \leq \int_{\left\{\lambda \in \sigma(A) \cap \mathcal{P}_{b, b_{+}}^{\beta} \mid \operatorname{Re} \lambda \geq 1\right\}} e^{s\left[\operatorname{Re} \lambda+b_{+}^{-\beta} \operatorname{Re} \lambda^{\beta}\right]^{1 / \beta}} e^{t \operatorname{Re} \lambda} \mathrm{d} v\left(f, g^{\star}, \lambda\right) \\
& \text { since, inview of } \operatorname{Re} \lambda \geq 1 \text { and } \beta \geq 1, \operatorname{Re} \lambda^{\beta} \geq \operatorname{Re} \lambda \text {; } \\
& \leq \int_{\left\{\lambda \in \sigma(A) \cap \mathscr{P} P_{b, b_{+}}^{\beta} \mid \operatorname{Re} \lambda \geq 1\right\}} e^{s\left(1+b_{+}^{-\beta}\right)^{1 / \beta} \operatorname{Re} \lambda} e^{t \operatorname{Re} \lambda} \mathrm{d} v\left(f, g^{\star}, \lambda\right) \\
& =\int_{\left\{\lambda \in \sigma(A) \cap \mathscr{P}_{b, b_{+}}^{\beta} \mid \operatorname{Re} \lambda \geq 1\right\}} e^{\left[s\left(1+b_{+}^{-\beta}\right)^{1 / \beta}+t\right] \operatorname{Re} \lambda} \mathrm{d} v\left(f, g^{\star}, \lambda\right) \\
& \text { since } f \in \bigcap D\left(e^{t A}\right) \text {, by Proposition 2.1; } \\
& <\infty \text {. }
\end{aligned}
$$

Finally, for any $s>0, t \in \mathbb{R}$ and an arbitrary $g^{\star} \in X^{\star}$,

$$
\begin{aligned}
& \int_{\left\{\lambda \in \sigma(A) \cap \mathscr{P}_{b_{-,} b_{+}}^{\beta} \mid \operatorname{Re} \lambda \leq-1\right\}} e^{s|\lambda|^{1 / \beta}} e^{t \operatorname{Re} \lambda} \mathrm{d} v\left(f, g^{\star}, \lambda\right) \\
& \leq \int_{\left\{\lambda \in \sigma(A) \cap \mathscr{P P}_{b, b_{+}}^{\beta} \mid \operatorname{Re} \lambda \leq-1\right\}} e^{s[|\operatorname{Re} \lambda|+|\operatorname{Im} \lambda|]^{1 / \beta}} e^{t \operatorname{Re} \lambda} \mathrm{d} v\left(f, g^{\star}, \lambda\right) \\
& \text { since, for } \lambda \in \Sigma(A) \cap \mathscr{P}_{b_{-,} b_{+}}^{\beta} \text { with } \operatorname{Re} \lambda \leq-1, b_{-}^{-\beta}(-\operatorname{Re} \lambda)^{\beta} \geq|\operatorname{Im} \lambda| \text {; } \\
& \leq \int_{\left\{\lambda \in \sigma(A) \cap \mathcal{P}_{b_{,}, b_{+}}^{\beta} \mid \operatorname{Re} \lambda \leq-1\right\}} e^{s\left[-\operatorname{Re} \lambda+b_{-}^{-\beta}(-\operatorname{Re} \lambda)^{\beta}\right]^{1 / \beta}} e^{t \operatorname{Re} \lambda} \mathrm{d} v\left(f, g^{\star}, \lambda\right) \\
& \text { since, in view of }-\operatorname{Re} \lambda \geq 1 \text { and } \beta \geq 1,(-\operatorname{Re} \lambda)^{\beta} \geq-\operatorname{Re} \lambda \text {; } \\
& \leq \int_{\left\{\lambda \in \sigma(A) \cap \mathscr{P}_{b_{-}, b_{+}}^{\beta} \mid \operatorname{Re} \lambda \leq-1\right\}} e^{S\left(1+b_{-}^{-\beta}\right)^{1 / \beta}(-\operatorname{Re} \lambda)} e^{t \operatorname{Re} \lambda} \mathrm{d} v\left(f, g^{\star}, \lambda\right) \\
& =\int_{\left\{\lambda \in \sigma(A) \cap \mathscr{P}_{b, b_{+}}^{\beta} \mid \operatorname{Re} \lambda \leq-1\right\}} e^{\left[t-s\left(1+b_{-}^{-\beta}\right)^{1 / \beta}\right] \operatorname{Re} \lambda} \mathrm{d} v\left(f, g^{\star}, \lambda\right)
\end{aligned}
$$$$
\text { since } f \in \bigcap_{t \in \mathbb{R}} D\left(e^{t A}\right) \text {, by Proposition 2.1; }
$$

$<\infty$.

Also, for any $s>0, t \in \mathbb{R}$ and an arbitrary $n \in \mathbb{N}$,

$$
\begin{aligned}
& \sup _{\left\{g^{\star} \in X^{\star} \mid\left\|g^{*}\right\|=1\right\}} \int_{\left\{\lambda \in \sigma(A) \mid e^{s|\lambda|^{1 / \beta}} e^{t \operatorname{Re} \lambda}>n\right\}} e^{s|\lambda|^{1 / \beta}} e^{t \operatorname{Re} \lambda} \mathrm{d} v\left(f, g^{\star}, \lambda\right) \\
& \quad \leq \sup _{\left\{g^{\star} \in X^{*} \mid\left\|g^{*}\right\|=1\right\}} \int_{\left\{\lambda \in \sigma(A)\left|\mathscr{P}_{b,-b_{+}}^{\beta}\right| e^{s \mid \lambda^{1 / \beta}} e^{t \operatorname{Re} \lambda}>n\right\}} e^{s|\lambda|^{1 / \beta}} e^{t \operatorname{Re} \lambda} \mathrm{d} v\left(f, g^{\star}, \lambda\right)
\end{aligned}
$$




$$
\begin{aligned}
& +\sup _{\left\{g^{\star} \in X^{\star} \mid\left\|g^{\star}\right\|=1\right\}} \int_{\substack{\left\{\lambda \in \sigma(A) \cap \mathscr{P}_{b-, b_{+}}^{\beta} \mid-1<\operatorname{Re} \lambda<1, e^{s|\lambda|^{1 / \beta}} e^{t \operatorname{Re} \lambda>n\}}\right.}} e^{s|\lambda|^{1 / \beta}} e^{t \operatorname{Re} \lambda} \mathrm{d} v\left(f, g^{\star}, \lambda\right) \\
& +\sup _{\left\{g^{\star} \in X^{\star} \mid\left\|g^{\star}\right\|=1\right\}} \int_{\left\{\lambda \in \sigma(A) \cap \mathscr{P}_{b_{-}, b_{+}}^{\beta} \mid \operatorname{Re} \lambda \geq 1, e^{s|\lambda|^{1 / \beta}} e^{t \operatorname{Re} \lambda>n\}}\right.} e^{s|\lambda|^{1 / \beta}} e^{t \operatorname{Re} \lambda} \mathrm{d} v\left(f, g^{\star}, \lambda\right) \\
& +\sup _{\left\{g^{*} \in X^{\star} \mid\left\|g^{\star}\right\|=1\right\}} \int_{\left\{\lambda \in \sigma(A) \cap \mathscr{P}_{b_{-}, b_{+}}^{\beta} \mid \operatorname{Re} \lambda \leq-1, e^{s|\lambda|^{1 / \beta}} e^{\left.t \operatorname{Re} \lambda_{>n}\right\}}\right.} e^{s|\lambda|^{1 / \beta}} e^{t \operatorname{Re} \lambda} \mathrm{d} v\left(f, g^{\star}, \lambda\right) \rightarrow 0, \quad n \rightarrow \infty .
\end{aligned}
$$

Indeed, since, due to the boundedness of the sets

$$
\sigma(A) \backslash \mathscr{P}_{b_{-}, b_{+}}^{\beta} \text { and } \quad\left\{\lambda \in \sigma(A) \cap \mathscr{P}_{b_{-}, b_{+}}^{\beta} \mid-1<\operatorname{Re} \lambda<1\right\}
$$

and the continuity of the integrated function on $\mathbb{C}$, the sets

$$
\left\{\lambda \in \sigma(A) \backslash \mathscr{P}_{b_{-}, b_{+}}^{\beta} \mid e^{s|\lambda|^{1 / \beta}} e^{t \operatorname{Re} \lambda}>n\right\}
$$

and

$$
\left\{\lambda \in \sigma(A) \cap \mathscr{P}_{b_{-}, b_{+}}^{\beta} \mid-1<\operatorname{Re} \lambda<1, e^{s|\lambda|^{1 / \beta}} e^{t \operatorname{Re} \lambda}>n\right\}
$$

are empty for all sufficiently large $n \in \mathbb{N}$, we immediately infer that, for any $s>0$ and $t \in \mathbb{R}$,

$$
\lim _{n \rightarrow \infty} \sup _{\left\{g^{\star} \in X^{\star} \mid\left\|g^{\star}\right\|=1\right\}} \int_{\substack{\left\{\lambda \in \sigma(A) \backslash \mathscr{P}_{b_{-}, b_{+}}^{\beta} \mid e^{s|\lambda|^{1 / \beta}} e^{\left.t \operatorname{Re} \lambda_{>n}\right\}}\\\\\right.}} e^{s|\lambda|^{1 / \beta}} e^{t \operatorname{Re} \lambda} \mathrm{d} v\left(f, g^{\star}, \lambda\right)=0
$$

and

$$
\lim _{n \rightarrow \infty} \sup _{\left\{g^{*} \in X^{\star} \mid\left\|g^{\star}\right\|=1\right\}} \int_{\left\{\lambda \in \sigma(A) \cap \mathscr{P}_{b_{-}, b_{+} \mid}^{\beta} \mid-1<\operatorname{Re} \lambda<1, e^{s|\lambda|^{1 / \beta}} e^{\left.t \operatorname{Re} \lambda_{>n}\right\}}\right.} e^{s|\lambda|^{1 / \beta}} e^{t \operatorname{Re} \lambda} \mathrm{d} v\left(f, g^{\star}, \lambda\right)=0 .
$$

Further, for any $s>0, t \in \mathbb{R}$ and an arbitrary $n \in \mathbb{N}$,

$$
\begin{aligned}
& \sup _{\left\{g^{\star} \in X^{\star} \mid\left\|g^{\star}\right\|=1\right\}} \int_{\left\{\lambda \in \sigma(A) \cap \mathscr{P}_{b_{-}, b_{+}}^{\beta} \mid \operatorname{Re} \lambda \geq 1, e^{s|\lambda|^{1 / \beta}} e^{\left.t \operatorname{Re} \lambda_{>n}\right\}}\right.} e^{s|\lambda|^{1 / \beta}} e^{t \operatorname{Re} \lambda} \mathrm{d} v\left(f, g^{\star}, \lambda\right) \\
& \leq \sup _{\left\{g^{\star} \in X^{\star} \mid\left\|g^{\star}\right\|=1\right\}} \int_{\substack{\left\{\lambda \in \sigma(A) \cap \mathscr{P}_{b_{-}, b_{+}}^{\beta} \mid \operatorname{Re} \lambda \geq 1, e^{s|\lambda|^{1 / \beta}} e^{t \operatorname{Re} \lambda>n\}}\\
\\
\right.}} e^{\left[s\left(1+b_{+}^{-\beta}\right)^{1 / \beta}+t\right] \operatorname{Re} \lambda} \mathrm{d} v\left(f, g^{\star}, \lambda\right) \\
& \text { since } f \in \bigcap_{t \in \mathbb{R}} D\left(e^{t A}\right) \text {, by (2.9); } \\
& \leq \sup _{\left\{g^{\star} \in X^{\star} \mid\left\|g^{\star}\right\|=1\right\}} 4 M\left\|E_{A}\left(\left\{\lambda \in \sigma(A) \cap \mathscr{P}_{b_{-}, b_{+}}^{\beta} \mid \operatorname{Re} \lambda \geq 1, e^{s|\lambda|^{1 / \beta}} e^{t \operatorname{Re} \lambda}>n\right\}\right) e^{\left[s\left(1+b_{+}^{-\beta}\right)^{1 / \beta}+t\right] A} f\right\|\left\|g^{\star}\right\| \\
& \leq 4 M\left\|E_{A}\left(\left\{\lambda \in \sigma(A) \cap \mathscr{P}_{b_{-}, b_{+}}^{\beta} \mid \operatorname{Re} \lambda \geq 1, e^{s|\lambda|^{1 / \beta}} e^{t \operatorname{Re} \lambda}>n\right\}\right) e^{\left[s\left(1+b_{+}^{-\beta}\right)^{1 / \beta}+t\right] A} f\right\| \\
& \text { by the strong continuity of the s.m.; } \\
& \rightarrow 4 M\left\|E_{A}(\varnothing) e^{\left[s\left(1+b_{+}^{-\beta}\right)^{1 / \beta}+t\right] A} f\right\|=0, \quad n \rightarrow \infty .
\end{aligned}
$$

Finally, for any $s>0, t \in \mathbb{R}$ and an arbitrary $n \in \mathbb{N}$,

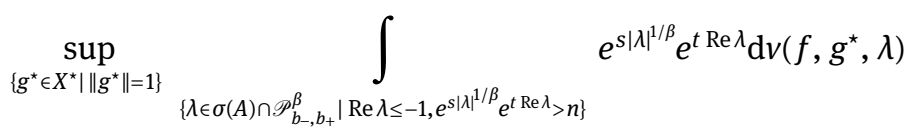




$$
\begin{aligned}
& \leq \sup _{\left\{g^{\star} \in X^{\star} \mid\left\|g^{\star}\right\|=1\right\}} \int_{\left\{\lambda \in \sigma(A) \cap \mathscr{P}_{b_{-}, b_{+}}^{\beta} \mid \operatorname{Re} \lambda \leq-1, e^{s|\lambda|^{1 / \beta}} e^{t \operatorname{Re} \lambda>n\}}\right.} e^{\left[t-s\left(1+b_{-}^{-\beta}\right)^{1 / \beta}\right] \operatorname{Re} \lambda} \mathrm{d} v\left(f, g^{\star}, \lambda\right) \\
& \leq \sup _{\left\{g^{\star} \in X^{\star} \mid\left\|g^{\star}\right\|=1\right\}} 4 M\left\|E_{A}\left(\left\{\lambda \in \sigma(A) \cap \mathscr{P}_{b_{-}, b_{+}}^{\beta} \mid \operatorname{Re} \lambda \geq 1, e^{s|\lambda|^{1 / \beta}} e^{t \operatorname{Re} \lambda}>n\right\}\right) e^{\left[t-s\left(1+b_{-}^{-\beta}\right)^{1 / \beta}\right] A} f\right\|\left\|g^{\star}\right\| \\
& \leq 4 M\left\|E_{A}\left(\left\{\lambda \in \sigma(A) \cap \mathscr{P}_{b_{-}, b_{+}}^{\beta} \mid \operatorname{Re} \lambda \geq 1, e^{s|\lambda|^{1 / \beta}} e^{t \operatorname{Re} \lambda}>n\right\}\right) e^{\left[t-s\left(1+b_{-}^{-\beta}\right)^{1 / \beta}\right] A} f\right\|
\end{aligned}
$$

by the strong continuity of the s.m.;

$$
\rightarrow 4 M\left\|E_{A}(\varnothing) e^{\left[t-s\left(1+b_{-}^{-\beta}\right)^{1 / \beta}\right] A} f\right\|=0, \quad n \rightarrow \infty .
$$

By Proposition 2.1 and the properties of the operational calculus (see [11, Theorem XVIII.2.11 (f)]), (4.16) and (4.19) jointly imply that, for any $s>0$ and $t \in \mathbb{R}$,

$$
f \in D\left(e^{S|A|^{1 / \beta}} e^{t A}\right),
$$

which, in view of (2.15), further implies that, for each $t \in \mathbb{R}$,

$$
y(t)=e^{t A} f \in \bigcap_{s>0} D\left(e^{s|A|^{1 / \beta}}\right)=\mathscr{E}^{(\beta)}(A) .
$$

Whence, by Proposition 3.1, we infer that

$$
y(\cdot) \in \mathscr{E}^{(\beta)}(\mathbb{R}, X),
$$

which completes the proof for the implication (iii) $\Rightarrow$ (i).

Let us prove the remaining implication (ii) $\Rightarrow$ (iii) by contrapositive assuming that, for any $b_{+}>0$ and $b_{-}>0$, the set $\sigma(A) \backslash \mathscr{P}_{b_{-}, b_{+}}^{\beta}$ is unbounded. In particular, this means that, for any $n \in \mathbb{N}$, unbounded is the set

$$
\sigma(A) \backslash \mathscr{P}_{n^{-1}, n^{-2}}^{\beta}=\left\{\left.\lambda \in \sigma(A)\left|-n^{-2}\right| \operatorname{Im} \lambda\right|^{1 / \beta}<\operatorname{Re} \lambda<n^{-2}|\operatorname{Im} \lambda|^{1 / \beta}\right\}
$$

Hence, we can choose a sequence $\left\{\lambda_{n}\right\}_{n=1}^{\infty}$ of points in the complex plane as follows:

$$
\begin{aligned}
& \lambda_{n} \in \sigma(A), \quad n \in \mathbb{N}, \\
& -n^{-2}\left|\operatorname{Im} \lambda_{n}\right|^{1 / \beta}<\operatorname{Re} \lambda_{n}<n^{-2}\left|\operatorname{Im} \lambda_{n}\right|^{1 / \beta}, \quad n \in \mathbb{N}, \\
& \lambda_{0}:=0,\left|\lambda_{n}\right|>\max \left[n,\left|\lambda_{n-1}\right|\right], \quad n \in \mathbb{N} .
\end{aligned}
$$

The latter implies, in particular, that the points $\lambda_{n}, n \in \mathbb{N}$, are $\operatorname{distinct}\left(\lambda_{i} \neq \lambda_{j}, i \neq j\right)$.

Since, for each $n \in \mathbb{N}$, the set

$$
\left\{\left.\lambda \in \mathbb{C}\left|-n^{-2}\right| \operatorname{Im} \lambda\right|^{1 / \beta}<\operatorname{Re} \lambda<n^{-2}|\operatorname{Im} \lambda|^{1 / \beta},|\lambda|>\max \left[n,\left|\lambda_{n-1}\right|\right]\right\}
$$

is open in $\mathbb{C}$, along with the point $\lambda_{n}$, it contains an open disk

$$
\Delta_{n}:=\left\{\lambda \in \mathbb{C}|| \lambda-\lambda_{n} \mid<\varepsilon_{n}\right\}
$$

centered at $\lambda_{n}$ of some radius $\varepsilon_{n}>0$, i.e., for each $\lambda \in \Delta_{n}$,

$$
-n^{-2}|\operatorname{Im} \lambda|^{1 / \beta}<\operatorname{Re} \lambda<n^{-2}|\operatorname{Im} \lambda|^{1 / \beta} \text { and }|\lambda|>\max \left[n,\left|\lambda_{n-1}\right|\right] .
$$

Furthermore, we can regard the radii of the disks to be small enough so that

$$
\begin{aligned}
& 0<\varepsilon_{n}<\frac{1}{n}, \quad n \in \mathbb{N}, \text { and } \\
& \Delta_{i} \cap \Delta_{j}=\varnothing, i \neq j \quad \text { (i.e., the disks are pairwise disjoint). }
\end{aligned}
$$

Whence, by the properties of the s.m.,

$$
E_{A}\left(\Delta_{i}\right) E_{A}\left(\Delta_{j}\right)=0, i \neq j,
$$

where 0 stands for the zero operator on $X$. 
Observe also that the subspaces $E_{A}\left(\Delta_{n}\right) X, n \in \mathbb{N}$, are nontrivial since

$$
\Delta_{n} \cap \sigma(A) \neq \varnothing, \quad n \in \mathbb{N},
$$

with $\Delta_{n}$ being an open set in $\mathbb{C}$.

In view of the pairwise disjointness of the disks $\Delta_{n}, n \in \mathbb{N}$, we can choose a unit vector $e_{n} \in E_{A}\left(\Delta_{n}\right) X$ for each $n \in \mathbb{N}$ obtaining a sequence $\left\{e_{n}\right\}_{n=1}^{\infty}$ in $X$ such that

$$
\left\|e_{n}\right\|=1, n \in \mathbb{N}, \quad \text { and } \quad E_{A}\left(\Delta_{i}\right) e_{j}=\delta_{i j} e_{j}, \quad i, j \in \mathbb{N},
$$

where $\delta_{i j}$ is the Kronecker delta.

As is easily seen, (4.22) implies that the vectors $e_{n}, n \in \mathbb{N}$, are linearly independent.

Furthermore, there exists an $\varepsilon>0$ such that

$$
d_{n}:=\operatorname{dist}\left(e_{n}, \operatorname{span}\left(\left\{e_{i} \mid i \in \mathbb{N}, i \neq n\right\}\right)\right) \geq \varepsilon, \quad n \in \mathbb{N} .
$$

Indeed, otherwise there exists a subsequence $\left\{d_{n(k)}\right\}_{k=1}^{\infty}$ such that

$$
d_{n(k)} \rightarrow 0, \quad k \rightarrow \infty \text {. }
$$

Then, by selecting a vector

$$
f_{n(k)} \in \operatorname{span}\left(\left\{e_{i} \mid i \in \mathbb{N}, i \neq n(k)\right\}\right), \quad k \in \mathbb{N},
$$

such that

$$
\left\|e_{n(k)}-f_{n(k)}\right\|<d_{n(k)}+1 / k, \quad k \in \mathbb{N},
$$

we arrive at

$$
\begin{aligned}
& 1=\left\|e_{n(k)}\right\| \\
& \text { since, by (4.22), } E_{A}\left(D_{n(k)}\right) e_{n(k)}=e_{n(k)} \text { and } E_{A}\left(D_{n(k)}\right) f_{n(k)}=0 \text {; } \\
& =\left\|E_{A}\left(\Delta_{n(k)}\right)\left(e_{n(k)}-f_{n(k)}\right)\right\| \leq\left\|E_{A}\left(\Delta_{n(k)}\right)\right\|\left\|e_{n(k)}-f_{n(k)}\right\| \quad \text { by (2.8); } \\
& \leq M\left\|e_{n(k)}-f_{n(k)}\right\| \leq M\left[d_{n(k)}+1 / k\right] \rightarrow 0, \quad k \rightarrow \infty,
\end{aligned}
$$

which is a contradiction proving (4.23).

As follows from the Hahn-Banach theorem, for any $n \in \mathbb{N}$, there is an $e_{n}^{\star} \in X^{\star}$ such that

$$
\left\|e_{n}^{\star}\right\|=1, n \in \mathbb{N}, \quad \text { and }\left\langle e_{i}, e_{j}^{\star}\right\rangle=\delta_{i j} d_{i}, i, j \in \mathbb{N} .
$$

Let us consider separately the two possibilities concerning the sequence of the real parts $\left\{\operatorname{Re} \lambda_{n\}_{n=1}^{\infty}}^{\infty}\right.$ : its being bounded or unbounded.

First, suppose that the sequence $\left\{\operatorname{Re} \lambda_{n}\right\}_{n=1}^{\infty}$ is bounded, i.e., there is such an $\omega>0$ that

$$
\left|\operatorname{Re} \lambda_{n}\right| \leq \omega, \quad n \in \mathbb{N},
$$

and consider the element

$$
f:=\sum_{k=1}^{\infty} k^{-2} e_{k} \in X
$$

which is well defined since $\left\{k^{-2}\right\}_{k=1}^{\infty} \in l_{1}\left(l_{1}\right.$ is the space of absolutely summable sequences) and $\left\|e_{k}\right\|=1$, $k \in \mathbb{N}$ (see (4.22)).

In view of (4.22), by the properties of the s.m.,

$$
E_{A}\left(\cup_{k=1}^{\infty} \Delta_{k}\right) f=f \quad \text { and } \quad E_{A}\left(\Delta_{k}\right) f=k^{-2} e_{k}, k \in \mathbb{N} .
$$

For any $t \geq 0$ and an arbitrary $g^{\star} \in X^{\star}$,

$$
\begin{aligned}
& \int_{\sigma(A)} e^{t \operatorname{Re} \lambda} \mathrm{d} v\left(f, g^{\star}, \lambda\right) \\
& \quad=\int_{\sigma(A)} e^{t \operatorname{Re} \lambda} \mathrm{d} v\left(E_{A}\left(\cup_{k=1}^{\infty} \Delta_{k}\right) f, g^{\star}, \lambda\right)
\end{aligned}
$$

by (2.12); 


$$
\begin{aligned}
& =\sum_{k=1}^{\infty} \int_{\sigma(A) \cap \Delta_{k}} e^{t \operatorname{Re} \lambda} \mathrm{d} v\left(E_{A}\left(\Delta_{k}\right) f, g^{\star}, \lambda\right) \\
& =\sum_{k=1}^{\infty} k^{-2} \int_{\sigma(A) \cap \Delta_{k}} e^{t \operatorname{Re} \lambda} \mathrm{d} v\left(e_{k}, g^{\star}, \lambda\right) \\
& \text { since, for } \lambda \in D_{k} \text {, by (4.25) and (4.21), } \\
& \operatorname{Re} \lambda=\operatorname{Re} \lambda_{k}+\left(\operatorname{Re} \lambda-\operatorname{Re} \lambda_{k}\right) \leq \operatorname{Re} \lambda_{k}+\left|\lambda-\lambda_{k}\right| \leq \omega+\varepsilon_{k} \leq \omega+1 ; \\
& \leq e^{t(\omega+1)} \sum_{k=1}^{\infty} k^{-2} \int_{\sigma(A) \cap \Delta_{k}} 1 \mathrm{~d} v\left(e_{k}, g^{\star}, \lambda\right)=e^{t(\omega+1)} \sum_{k=1}^{\infty} k^{-2} v\left(e_{k}, g^{\star}, \Delta_{k}\right) \\
& \leq e^{t(\omega+1)} \sum_{k=1}^{\infty} k^{-2} 4 M\left\|e_{k}\right\|\left\|g^{\star}\right\|=4 M e^{t(\omega+1)}\left\|g^{\star}\right\| \sum_{k=1}^{\infty} k^{-2}<\infty .
\end{aligned}
$$

by $(2.10)$;

Also, for any $t<0$ and an arbitrary $g^{\star} \in X^{\star}$,

$$
\begin{array}{rlr}
\int_{\sigma(A)} e^{t} & \operatorname{Re} \lambda \mathrm{d} v\left(f, g^{\star}, \lambda\right) & \text { by (4.26); } \\
& =\int_{\sigma(A)} e^{t \operatorname{Re} \lambda} \mathrm{d} v\left(E_{A}\left(\cup_{k=1}^{\infty} \Delta_{k}\right) f, g^{\star}, \lambda\right) & \text { by (2.12); } \\
& =\sum_{k=1}^{\infty} \int_{\sigma(A) \cap \Delta_{k}} e^{t \operatorname{Re} \lambda} \mathrm{d} v\left(E_{A}\left(\Delta_{k}\right) f, g^{\star}, \lambda\right) & \text { by (4.26); } \\
& =\sum_{k=1}^{\infty} k^{-2} \int_{\sigma(A) \cap \Delta_{k}} e^{t \operatorname{Re} \lambda} \mathrm{d} v\left(e_{k}, g^{\star}, \lambda\right) &
\end{array}
$$

since, for $\lambda \in D_{k}$, by (4.25) and (4.21),

$$
\begin{aligned}
& \operatorname{Re} \lambda=\operatorname{Re} \lambda_{k}-\left(\operatorname{Re} \lambda_{k}-\operatorname{Re} \lambda\right) \geq \operatorname{Re} \lambda_{k}-\left|\operatorname{Re} \lambda_{k}-\operatorname{Re} \lambda\right| \geq-\omega-\varepsilon_{k} \geq-\omega-1 ; \\
& \leq e^{-t(\omega+1)} \sum_{k=1}^{\infty} k^{-2} \int_{\sigma(A) \cap \Delta_{k}} 1 \mathrm{~d} v\left(e_{k}, g^{\star}, \lambda\right)=e^{-t(\omega+1)} \sum_{k=1}^{\infty} k^{-2} v\left(e_{k}, g^{\star}, \Delta_{k}\right)
\end{aligned}
$$$$
\leq e^{-t(\omega+1)} \sum_{k=1}^{\infty} k^{-2} 4 M\left\|e_{k}\right\|\left\|g^{\star}\right\|=4 M e^{-t(\omega+1)}\left\|g^{\star}\right\| \sum_{k=1}^{\infty} k^{-2}<\infty .
$$

Similar to (4.27) for any $t \geq 0$ and an arbitrary $n \in \mathbb{N}$,

$$
\begin{aligned}
& \sup _{\left\{g^{\star} \in X^{\star} \mid\left\|g^{\star}\right\|=1\right\}} \int_{\left\{\lambda \in \sigma(A) \mid e^{t \operatorname{Re} \lambda>n\}}\right.} e^{t \operatorname{Re} \lambda} \mathrm{d} v\left(f, g^{\star}, \lambda\right) \\
& \leq \sup _{\left\{g^{\star} \in X^{\star} \mid\left\|g^{\star}\right\|=1\right\}} e^{t(\omega+1)} \sum_{k=1}^{\infty} k^{-2} \int_{\left\{\lambda \in \sigma(A) \mid e^{t \operatorname{Re} \lambda>n\} \cap \Delta_{k}}\right.} 1 \mathrm{~d} v\left(e_{k}, g^{\star}, \lambda\right) \\
& =e^{t(\omega+1)} \sup _{\left\{g^{\star} \in X^{\star} \mid\left\|g^{\star}\right\|=1\right\}} \sum_{k=1}^{\infty} \int_{\left\{\lambda \in \sigma(A) \mid e^{t \operatorname{Re} \lambda}>n\right\} \cap \Delta_{k}} 1 \mathrm{~d} v\left(E_{A}\left(\Delta_{k}\right) f, g^{\star}, \lambda\right) \\
& =e^{t(\omega+1)} \sup _{\left\{g^{\star} \in X^{\star} \mid\left\|g^{\star}\right\|=1\right\}} \int_{\left\{\lambda \in \sigma(A) \mid e^{t \operatorname{Re} \lambda>n\}}\right.} 1 \mathrm{~d} v\left(E_{A}\left(\cup_{k=1}^{\infty} \Delta_{k}\right) f, g^{\star}, \lambda\right) \\
& =e^{t(\omega+1)} \sup _{\left\{g^{\star} \in X^{\star} \mid\left\|g^{\star}\right\|=1\right\}} \int_{\left\{\lambda \in \sigma(A) \mid e^{t \operatorname{Re} \lambda>n\}}\right.} 1 \mathrm{~d} v\left(f, g^{\star}, \lambda\right)
\end{aligned}
$$$$
\text { by (4.26); }
$$

by (2.12); 


$$
\begin{aligned}
& \leq e^{t(\omega+1)} \sup _{\left\{g^{\star} \in X^{\star} \mid\left\|g^{\star}\right\|=1\right\}} 4 M\left\|E_{A}\left(\left\{\lambda \in \sigma(A) \mid e^{t \operatorname{Re} \lambda}>n\right\}\right) f\right\|\left\|g^{\star}\right\| \\
& \leq 4 M e^{t(\omega+1)}\left\|E_{A}\left(\left\{\lambda \in \sigma(A) \mid e^{t \operatorname{Re} \lambda}>n\right\}\right) f\right\| \\
& \rightarrow 4 M e^{t(\omega+1)}\left\|E_{A}(\varnothing) f\right\|=0, \quad n \rightarrow \infty .
\end{aligned}
$$

Similar to (4.28) for any $t<0$ and an arbitrary $n \in \mathbb{N}$,

$$
\begin{aligned}
& \sup _{\left\{g^{\star} \in X^{\star} \mid\left\|g^{*}\right\|=1\right\}} \int_{\left\{\lambda \in \sigma(A) \mid e^{t \operatorname{Re} \lambda}>n\right\}} e^{t \operatorname{Re} \lambda} \mathrm{d} v\left(f, g^{\star}, \lambda\right) \\
& \leq \sup _{\left\{g^{*} \in X^{\star}\left\|g^{\star}\right\|=1\right\}} e^{-t(\omega+1)} \sum_{k=1}^{\infty} k^{-2} \int_{\left\{\lambda \in \sigma(A) \mid e^{t \operatorname{Re} \lambda}>n\right\} \cap \Delta_{k}} 1 \mathrm{~d} v\left(e_{k}, g^{\star}, \lambda\right) \\
& =e^{-t(\omega+1)} \sup _{\left\{g^{*} \in X^{\star} \mid\left\|g^{\star}\right\|=1\right\}} \sum_{k=1}^{\infty} \int_{\left\{\lambda \in \sigma(A) \mid e^{t \mathrm{Re} \lambda}>n\right\} \cap \Delta_{k}} 1 \mathrm{~d} v\left(E_{A}\left(\Delta_{k}\right) f, g^{\star}, \lambda\right) \\
& =e^{-t(\omega+1)} \sup _{\left\{g^{\star} \in X^{\star} \mid\left\|g^{\star}\right\|=1\right\}} \int_{\left\{\lambda \in \sigma(A) \mid e^{t \operatorname{Re} \lambda}>n\right\}} 1 \mathrm{~d} v\left(E_{A}\left(\cup_{k=1}^{\infty} \Delta_{k}\right) f, g^{\star}, \lambda\right) \\
& =e^{-t(\omega+1)} \sup _{\left\{g^{\star} \in X^{\star} \mid\left\|g^{\star}\right\|=1\right\}} \int_{\left\{\lambda \in \sigma(A) \mid e^{t \operatorname{Re} \lambda>n\}}\right.} 1 \mathrm{~d} v\left(f, g^{\star}, \lambda\right) \\
& \leq e^{-t(\omega+1)} \sup _{\left\{g^{*} \in X^{\star}\left\|g^{\star}\right\|=1\right\}} 4 M\left\|E_{A}\left(\left\{\lambda \in \sigma(A) \mid e^{t \operatorname{Re} \lambda}>n\right\}\right) f\right\|\left\|g^{\star}\right\| \\
& \leq 4 M e^{-t(\omega+1)}\left\|E_{A}\left(\left\{\lambda \in \sigma(A) \mid e^{t \operatorname{Re} \lambda}>n\right\}\right) f\right\| \\
& \rightarrow 4 M e^{-t(\omega+1)}\left\|E_{A}(\varnothing) f\right\|=0, n \rightarrow \infty .
\end{aligned}
$$

By Proposition 2.1, (4.27), (4.28), (4.29), and (4.30) jointly imply that

$$
f \in \bigcap_{t \in \mathbb{R}} D\left(e^{t A}\right),
$$

and hence, by Theorem 2.1,

$$
y(t):=e^{t A} f, \quad t \in \mathbb{R},
$$

is a weak solution of equation (1.1).

Let

$$
h^{\star}:=\sum_{k=1}^{\infty} k^{-2} e_{k}^{\star} \in X^{\star},
$$

the functional being well defined since $\left\{k^{-2}\right\}_{k=1}^{\infty} \in l_{1}$ and $\left\|e_{k}^{\star}\right\|=1, k \in \mathbb{N}$ (see (4.24)).

In view of (4.24) and (4.23), we have:

$$
\left\langle e_{n}, h^{\star}\right\rangle=\left\langle e_{k}, k^{-2} e_{k}^{\star}\right\rangle=d_{k} k^{-2} \geq \varepsilon k^{-2}, \quad k \in \mathbb{N} .
$$

For any $s>0$,

$$
\begin{array}{rr}
\int_{\sigma(A)} e^{s|\lambda|^{1 / \beta}} \mathrm{d} v\left(f, h^{\star}, \lambda\right) & \text { by (2.12) as in (4.27); } \\
=\sum_{k=1}^{\infty} k^{-2} \int_{\sigma(A) \cap \Delta_{k}} e^{s|\lambda|^{1 / \beta}} \mathrm{d} v\left(e_{k}, h^{\star}, \lambda\right) & \text { since, for } \lambda \in D_{k} \text {, by (4.20), }|\lambda| \geq k ;
\end{array}
$$




$$
\begin{array}{ll}
\geq \sum_{k=1}^{\infty} k^{-2} e^{s k^{1 / \beta}} \int_{\sigma(A) \cap \Delta_{k}} 1 \mathrm{~d} v\left(e_{k}, h^{\star}, \lambda\right)=\sum_{k=1}^{\infty} k^{-2} e^{s k^{1 / \beta}} v\left(e_{k}, h^{\star}, \Delta_{k}\right) \\
\geq \sum_{k=1}^{\infty} k^{-2} e^{s k^{1 / \beta}\left|\left\langle E_{A}\left(\Delta_{k}\right) e_{k}, h^{\star}\right\rangle\right|} \\
\geq \sum_{k=1}^{\infty} \varepsilon k^{-4} e^{s k^{1 / \beta}}=\infty & \text { by (4.22) and (4.32); }
\end{array}
$$

Whence, by Proposition 2.1 and (2.15), we infer that

$$
y(0)=f \notin \bigcup_{s>0} D\left(e^{s|A|^{1 / \beta}}\right)=\mathscr{E}^{\{\beta\}}(A),
$$

which, by Proposition 3.1, implies that the weak solution $y(t)=e^{t A} f, t \in \mathbb{R}$, of equation (1.1) does not belong to the Roumieu-type Gevrey class $\mathscr{E}^{\{\beta\}}(\mathbb{R}, X)$ and completes our consideration of the case of the sequence's $\left\{\operatorname{Re} \lambda_{n}\right\}_{n=1}^{\infty}$ being bounded.

Now, suppose that the sequence $\left\{\operatorname{Re} \lambda_{n}\right\}_{n=1}^{\infty}$ is unbounded.

Therefore, there is a subsequence $\left\{\operatorname{Re} \lambda_{n(k)}\right\}_{k=1}^{\infty}$ such that

$$
\operatorname{Re} \lambda_{n(k)} \rightarrow \infty \quad \text { or } \quad \operatorname{Re} \lambda_{n(k)} \rightarrow-\infty, \quad k \rightarrow \infty .
$$

Let us consider separately each of the two cases.

First, suppose that

$$
\operatorname{Re} \lambda_{n(k)} \rightarrow \infty, \quad k \rightarrow \infty
$$

Then, without loss of generality, we can regard that

$$
\operatorname{Re} \lambda_{n(k)} \geq k, \quad k \in \mathbb{N} .
$$

Consider the elements

$$
f:=\sum_{k=1}^{\infty} e^{-n(k) \operatorname{Re} \lambda_{n(k)}} e_{n(k)} \in X \quad \text { and } \quad h:=\sum_{k=1}^{\infty} e^{-\frac{n(k)}{2} \operatorname{Re} \lambda_{n(k)}} e_{n(k)} \in X,
$$

well defined since, by (4.33),

$$
\left\{e^{-n(k) \operatorname{Re} \lambda_{n(k)}}\right\}_{k=1}^{\infty},\left\{e^{-\frac{n(k)}{2} \operatorname{Re} \lambda_{n(k)}}\right\}_{k=1}^{\infty} \in l_{1}
$$

and $\left\|e_{n(k)}\right\|=1, k \in \mathbb{N}$ (see (4.22)).

By (4.22),

$$
E_{A}\left(\bigcup_{k=1}^{\infty} \Delta_{n(k)}\right) f=f \quad \text { and } \quad E_{A}\left(\Delta_{n(k)}\right) f=e^{-n(k) \operatorname{Re} \lambda_{n(k)}} e_{n(k)}, \quad k \in \mathbb{N} \text {, }
$$

and

$$
E_{A}\left(\bigcup_{k=1}^{\infty} \Delta_{n(k)}\right) h=h \quad \text { and } \quad E_{A}\left(\Delta_{n(k)}\right) h=e^{-\frac{n(k)}{2} \operatorname{Re} \lambda_{n(k)}} e_{n(k)}, \quad k \in \mathbb{N} .
$$

For any $t \geq 0$ and an arbitrary $g^{\star} \in X^{\star}$,

$$
\begin{aligned}
& \int_{\sigma(A)} e^{t \operatorname{Re} \lambda} \mathrm{d} v\left(f, g^{\star}, \lambda\right) \quad \text { by (2.12) as in (4.27); } \\
& =\sum_{k=1}^{\infty} e^{-n(k) \operatorname{Re} \lambda_{n(k)}} \int_{\sigma(A) \cap \Delta_{n(k)}} e^{t \operatorname{Re} \lambda} \mathrm{d} v\left(e_{n(k)}, g^{\star}, \lambda\right) \\
& \text { since, for } \lambda \in \Delta_{n(k)} \text {, by (4.21), } \\
& \operatorname{Re} \lambda=\operatorname{Re} \lambda_{n(k)}+\left(\operatorname{Re} \lambda-\operatorname{Re} \lambda_{n(k)}\right) \leq \operatorname{Re} \lambda_{n(k)}+\left|\lambda-\lambda_{n(k)}\right| \leq \operatorname{Re} \lambda_{n(k)}+1 ; \\
& \leq \sum_{k=1}^{\infty} e^{-n(k) \operatorname{Re} \lambda_{n(k)}} e^{t\left(\operatorname{Re} \lambda_{n(k)}+1\right)} \int_{\sigma(A) \cap \Delta_{n(k)}} 1 \mathrm{~d} v\left(e_{n(k)}, g^{\star}, \lambda\right)
\end{aligned}
$$




$$
\begin{aligned}
& =e^{t} \sum_{k=1}^{\infty} e^{-[n(k)-t] \operatorname{Re} \lambda_{n(k)}} v\left(e_{n(k)}, g^{\star}, \Delta_{n(k)}\right) \\
& \leq e^{t} \sum_{k=1}^{\infty} e^{-[n(k)-t] \operatorname{Re} \lambda_{n(k)}} 4 M\left\|e_{n(k)}\right\|\left\|g^{\star}\right\|=4 M e^{t}\left\|g^{\star}\right\| \sum_{k=1}^{\infty} e^{-[n(k)-t] \operatorname{Re} \lambda_{n(k)}} \\
& <\infty .
\end{aligned}
$$

Indeed, for all $k \in \mathbb{N}$ sufficiently large so that

$$
n(k) \geq t+1
$$

in view of (4.33),

$$
e^{-[n(k)-t] \operatorname{Re} \lambda_{n(k)} \leq e^{-k}}
$$

For any $t<0$ and an arbitrary $g^{\star} \in X^{\star}$,

$$
\begin{aligned}
& \int_{\sigma(A)} e^{t \operatorname{Re} \lambda} \mathrm{d} v\left(f, g^{\star}, \lambda\right) \quad \text { by (2.12) as in (4.27); } \\
& =\sum_{k=1}^{\infty} e^{-n(k) \operatorname{Re} \lambda_{n(k)}} \int_{\sigma(A) \cap \Delta_{n(k)}} e^{t \operatorname{Re} \lambda} \mathrm{d} v\left(e_{n(k)}, g^{\star}, \lambda\right) \\
& \text { since, for } \lambda \in \Delta_{n(k)} \text {, by (4.21), } \\
& \operatorname{Re} \lambda=\operatorname{Re} \lambda_{n(k)}-\left(\operatorname{Re} \lambda_{n(k)}-\operatorname{Re} \lambda\right) \geq \operatorname{Re} \lambda_{n(k)}-\left|\operatorname{Re} \lambda_{n(k)}-\operatorname{Re} \lambda\right| \geq \operatorname{Re} \lambda_{n(k)}-1 ; \\
& \leq \sum_{k=1}^{\infty} e^{-n(k) \operatorname{Re} \lambda_{n(k)}} e^{t\left(\operatorname{Re} \lambda_{n(k)}-1\right)} \int_{\sigma(A) \cap \Delta_{n(k)}} 1 \mathrm{~d} v\left(e_{n(k)}, g^{\star}, \lambda\right) \\
& =e^{-t} \sum_{k=1}^{\infty} e^{-[n(k)-t] \operatorname{Re} \lambda_{n(k)}} v\left(e_{n(k)}, g^{\star}, \Delta_{n(k)}\right) \\
& \leq e^{-t} \sum_{k=1}^{\infty} e^{-[n(k)-t] \operatorname{Re} \lambda_{n(k)}} 4 M\left\|e_{n(k)}\right\|\left\|g^{\star}\right\|=4 M e^{-t}\left\|g^{\star}\right\| \sum_{k=1}^{\infty} e^{-[n(k)-t] \operatorname{Re} \lambda_{n(k)}} \\
& <\infty \text {. }
\end{aligned}
$$

Indeed, for all $k \in \mathbb{N}$, in view of $t<0$,

$$
n(k)-t \geq n(k) \geq 1 \text {, }
$$

and hence, in view of (4.33),

$$
e^{-[n(k)-t] \operatorname{Re} \lambda_{n(k)}} \leq e^{-k} .
$$

Similarly to (4.36), for any $t \geq 0$ and an arbitrary $n \in \mathbb{N}$,

$$
\begin{aligned}
& \sup _{\left\{g^{\star} \in X^{\star} \mid\left\|g^{\star}\right\|=1\right\}} \int_{\left\{\lambda \in \sigma(A) \mid e^{t \operatorname{Re} \lambda}>n\right\}} e^{t \operatorname{Re} \lambda} \mathrm{d} v\left(f, g^{\star}, \lambda\right) \\
& \leq \sup _{\left\{g^{\star} \in X^{\star} \mid\left\|g^{\star}\right\|=1\right\}} e^{t} \sum_{k=1}^{\infty} e^{-[n(k)-t] \operatorname{Re} \lambda_{n(k)}} \int_{\left\{\lambda \in \sigma(A) \mid e^{\left.t \operatorname{Re} \lambda_{>n}\right\} \cap \Delta_{n(k)}}\right.} 1 \mathrm{~d} v\left(e_{n(k)}, g^{\star}, \lambda\right) \\
& =e^{t} \sup _{\left\{g^{\star} \in X^{\star} \mid\left\|g^{\star}\right\|=1\right\}} \sum_{k=1}^{\infty} e^{-\left[\frac{n(k)}{2}-t\right] \operatorname{Re} \lambda_{n(k)}} e^{-\frac{n(k)}{2} \operatorname{Re} \lambda_{(k)}} \int_{\left\{\lambda \in \sigma(A) \mid e^{t \operatorname{Re} \lambda_{>}}{ }_{n\} \cap \Delta_{n(k)}}\right.} 1 \mathrm{~d} v\left(e_{n(k)}, g^{\star}, \lambda\right) \\
& \text { since, by (4.33), there is an } L>0 \text { such that } e^{-\left[\frac{n(k)}{2}-t\right] \operatorname{Re} l_{n(k)}} \leq L, k \in \mathbb{N} \text {; } \\
& \leq L e^{t} \sup _{\left\{g^{*} \in X^{\star} \mid\left\|g^{\star}\right\|=1\right\}} \sum_{k=1}^{\infty} e^{-\frac{n(k)}{2} \operatorname{Re} \lambda_{n(k)}} \int_{\left\{\lambda \in \sigma(A) \mid e^{t \operatorname{Re} \lambda>n\} \cap \Delta_{n(k)}}\right.} 1 \mathrm{~d} v\left(e_{n(k)}, g^{\star}, \lambda\right)
\end{aligned}
$$




$$
\begin{array}{llr} 
& =L e^{t} \sup _{\left\{g^{\star} \in X^{\star} \mid\left\|g^{\star}\right\|=1\right\}} \int_{\left\{\lambda \in \sigma(A) \mid e^{t \operatorname{Re} \lambda>n\}}\right.} 1 \mathrm{~d} v\left(E_{A}\left(\cup_{k=1}^{\infty} \Delta_{n(k)}\right) h, g^{\star}, \lambda\right) & \text { by (2.12); } \\
=L e^{t} \sup _{\left\{g^{\star} \in X^{\star} \mid\left\|g^{\star}\right\|=1\right\}} \int_{\left\{\lambda \in \sigma(A) \mid e^{\left.t \operatorname{Re} \lambda_{>}\right\}}\right.} 1 \mathrm{~d} v\left(h, g^{\star}, \lambda\right) & \text { by (2.12); } \\
& \int_{\left\{g^{t} \in X^{\star} \mid\left\|g^{\star}\right\|=1\right\}} \int_{\left\{\lambda \in \sigma(A) \mid e^{t \operatorname{Re} \lambda>n\}}\right.} 1 \mathrm{~d} v\left(h, g^{\star}, \lambda\right) & \text { by (4.35); } \\
\leq L e^{t} \sup _{\left\{g^{\star} \in X^{\star} \mid\left\|g^{\star}\right\|=1\right\}} 4 M\left\|E_{A}\left(\left\{\lambda \in \sigma(A) \mid e^{t \operatorname{Re} \lambda}>n\right\}\right) h\right\|\left\|g^{\star}\right\| & \text { by (2.9); } \\
\leq 4 L M e^{t}\left\|E_{A}\left(\left\{\lambda \in \sigma(A) \mid e^{t \operatorname{Re} \lambda}>n\right\}\right) h\right\| &
\end{array}
$$$$
\rightarrow 4 L M e^{t}\left\|E_{A}(\varnothing) h\right\|=0, n \rightarrow \infty
$$

by the strong continuity of the s.m.;

Similarly to (4.37), for any $t<0$ and an arbitrary $n \in \mathbb{N}$,

$$
\begin{aligned}
& \sup _{\left\{g^{\star} \in X^{\star} \mid\left\|g^{\star}\right\|=1\right\}} \int_{\left\{\lambda \in \sigma(A) \mid e^{\left.t \operatorname{Re} \lambda_{>}\right\}}\right.} e^{t \operatorname{Re} \lambda} \mathrm{d} v\left(f, g^{\star}, \lambda\right) \\
& \leq \sup _{\left\{g^{\star} \in X^{\star} \mid\left\|g^{\star}\right\|=1\right\}} e^{-t} \sum_{k=1}^{\infty} e^{-[n(k)-t] \operatorname{Re} \lambda_{n(k)}} \int_{\left\{\lambda \in \sigma(A) \mid e^{t \operatorname{Re} \lambda_{>}}, n\right\} \cap \Delta_{n(k)}} 1 \mathrm{~d} v\left(e_{n(k)}, g^{\star}, \lambda\right) \\
& =e^{-t} \sup _{\left\{g^{\star} \in X^{\star} \mid\left\|g^{*}\right\|=1\right\}} \sum_{k=1}^{\infty} e^{-\left[\frac{n(k)}{2}-t\right] \operatorname{Re} \lambda_{n(k)}} e^{-\frac{n(k)}{2} \operatorname{Re} \lambda_{(k)}} \int_{\left\{\lambda \in \sigma(A) \mid e^{t \operatorname{Re} \lambda_{>}}{ }_{n\} \cap \Delta_{n(k)}}\right.} 1 \mathrm{~d} v\left(e_{n(k)}, g^{\star}, \lambda\right)
\end{aligned}
$$

since, by (4.33), there is an $L>0$ such that $e^{-\left[\frac{n(k)}{2}-t\right] \operatorname{Re} \lambda_{n(k)}} \leq L, k \in \mathbb{N}$;

$$
\leq L e^{-t} \sup _{\left\{g^{\star} \in X^{\star} \mid\left\|g^{\star}\right\|=1\right\}} \sum_{k=1}^{\infty} e^{-\frac{n(k)}{2} \operatorname{Re} \lambda_{n(k)}} \int_{\left\{\lambda \in \sigma(A) \mid e^{t \operatorname{Re} \lambda_{>}}>n \cap \Delta_{n(k)}\right.} 1 \mathrm{~d} v\left(e_{n(k)}, g^{\star}, \lambda\right)
$$
by (4.35);

$$
\begin{aligned}
& =L e^{-t} \sup _{\left\{g^{\star} \in X^{\star} \mid\left\|g^{\star}\right\|=1\right\}} \sum_{k=1}^{\infty} \int_{\left\{\lambda \in \sigma(A) \mid e^{t \operatorname{Re} \lambda}>n\right\} \cap \Delta_{n(k)}} 1 \mathrm{~d} v\left(E_{A}\left(\Delta_{n(k)}\right) h, g^{\star}, \lambda\right) \\
& =L e^{-t} \sup _{\left\{g^{\star} \in X^{\star} \mid\left\|g^{\star}\right\|=1\right\}} \int_{\left\{\lambda \in \sigma(A) \mid e^{t \operatorname{Re} \lambda>n\}}\right.} 1 \mathrm{~d} v\left(E_{A}\left(\cup_{k=1}^{\infty} \Delta_{n(k)}\right) h, g^{\star}, \lambda\right) \\
& =L e^{-t} \sup _{\left\{g^{\star} \in X^{\star} \mid\left\|g^{\star}\right\|=1\right\}} \int_{\left\{\lambda \in \sigma(A) \mid e^{t \operatorname{Re} \lambda}>n\right\}} 1 \mathrm{~d} v\left(h, g^{\star}, \lambda\right) \\
& \leq L e^{-t} \sup _{\left\{g^{\star} \in X^{\star} \mid\left\|g^{\star}\right\|=1\right\}} 4 M\left\|E_{A}\left(\left\{\lambda \in \sigma(A) \mid e^{t \operatorname{Re} \lambda}>n\right\}\right) h\right\|\left\|g^{\star}\right\| \\
& \leq 4 L M e^{-t}\left\|E_{A}\left(\left\{\lambda \in \sigma(A) \mid e^{t \operatorname{Re} \lambda}>n\right\}\right) h\right\|
\end{aligned}
$$$$
\rightarrow 4 L M e^{-t}\left\|E_{A}(\varnothing) h\right\|=0, \quad n \rightarrow \infty
$$

by (2.12);

by (4.35);

by (2.9);

By Proposition 2.1, (4.36), (4.37), (4.38), and (4.39) jointly imply that

$$
f \in \bigcap_{t \in \mathbb{R}} D\left(e^{t A}\right),
$$

and hence, by Theorem 2.1,

$$
y(t):=e^{t A} f, \quad t \in \mathbb{R},
$$

is a weak solution of equation (1.1). 
Since, for any $\lambda \in \Delta_{n(k)}, k \in \mathbb{N}$, by (4.21), (4.33),

$$
\begin{aligned}
\operatorname{Re} \lambda & =\operatorname{Re} \lambda_{n(k)}-\left(\operatorname{Re} \lambda_{n(k)}-\operatorname{Re} \lambda\right) \geq \operatorname{Re} \lambda_{n(k)}-\left|\operatorname{Re} \lambda_{n(k)}-\operatorname{Re} \lambda\right| \\
& \geq \operatorname{Re} \lambda_{n(k)}-\varepsilon_{n(k)} \geq \operatorname{Re} \lambda_{n(k)}-1 / n(k) \geq k-1 \geq 0
\end{aligned}
$$

and, by (4.20),

$$
\operatorname{Re} \lambda<n(k)^{-2}|\operatorname{Im} \lambda|^{1 / \beta},
$$

we infer that, for any $\lambda \in \Delta_{n(k)}, k \in \mathbb{N}$,

$$
|\lambda| \geq|\operatorname{Im} \lambda| \geq\left[n(k)^{2} \operatorname{Re} \lambda\right]^{\beta} \geq\left[n(k)^{2}\left(\operatorname{Re} \lambda_{n(k)}-1 / n(k)\right)\right]^{\beta} .
$$

Using this estimate, for an arbitrary $s>0$ and the functional $h^{\star} \in X^{\star}$ defined by (4.31), we have:

$$
\begin{aligned}
\int_{\sigma(A)} e^{s|\lambda|^{1 / \beta}} \mathrm{d} v\left(f, h^{\star}, \lambda\right) & \\
\quad & \sum_{k=1}^{\infty} e^{-n(k) \operatorname{Re} \lambda_{n(k)}} \int_{\sigma(A) \cap \Delta_{n(k)}} e^{s|\lambda|^{1 / \beta}} \mathrm{d} v\left(e_{n(k)}, h^{\star}, \lambda\right) \\
& \geq \sum_{k=1}^{\infty} e^{-n(k) \operatorname{Re} \lambda_{n(k)}} e^{s n(k)^{2}\left(\operatorname{Re} \lambda_{n(k)}-1 / n(k)\right)} v\left(e_{n(k)}, h^{\star}, \Delta_{n(k)}\right) \\
& \geq \sum_{k=1}^{\infty} e^{-n(k) \operatorname{Re} \lambda_{n(k)}} e^{s n(k)^{2}\left(\operatorname{Re} \lambda_{n(k)}-1 / n(k)\right)}\left|\left\langle E_{A}\left(\Delta_{n(k)}\right) e_{n(k)}, h^{\star}\right\rangle\right| \\
& \geq \sum_{k=1}^{\infty} \varepsilon e^{(s n(k)-1) n(k) \operatorname{Re} \lambda_{n(k)}-s n(k)} n(k)^{-2}=\infty .
\end{aligned}
$$

by (2.12) as in (4.27);

Indeed, for all $k \in \mathbb{N}$ sufficiently large so that

$$
\operatorname{sn}(k) \geq s+2,
$$

in view of (4.33),

$$
e^{(s n(k)-1) n(k) \operatorname{Re} \lambda_{n(k)}-s n(k)} n(k)^{-2} \geq e^{(s+1) n(k)-s n(k)} n(k)^{-2}=e^{n(k)} n(k)^{-2} \rightarrow \infty, \quad k \rightarrow \infty .
$$

Whence, by Proposition 2.1 and (2.15), we infer that

$$
y(0)=f \notin \bigcup_{s>0} D\left(e^{s|A|^{1 / \beta}}\right)=\mathscr{E}^{\{\beta\}}(A),
$$

which, by Proposition 3.1, implies that the weak solution $y(t)=e^{t A} f, t \in \mathbb{R}$, of equation (1.1) does not belong to the Roumieu-type Gevrey class $\left.\mathscr{E}^{\{}\{\}\right\}(\mathbb{R}, X)$.

The remaining case of

$$
\operatorname{Re} \lambda_{n(k)} \rightarrow-\infty, \quad k \rightarrow \infty,
$$

is symmetric to the case of

$$
\operatorname{Re} \lambda_{n(k)} \rightarrow \infty, \quad k \rightarrow \infty,
$$

and is considered in absolutely the same manner, which furnishes a weak solution $y(\cdot)$ of equation (1.1) such that

$$
y(0)=f \notin \bigcup_{s>0} D\left(e^{s|A|^{1 / \beta}}\right)=\mathscr{E}^{\{\beta\}}(A),
$$

and hence, by Proposition 3.1, not belonging to the Roumieu-type Gevrey class $\mathscr{E}\{\beta\}(\mathbb{R}, X)$.

With every possibility concerning $\left\{\operatorname{Re} \lambda_{n}\right\}_{n=1}^{\infty}$ considered, the proof by contrapositive of the "only if" part is complete and so is the proof of the entire statement. 
Remark 4.1. Due to the scalar type spectrality of the operator $A$, Theorem 4.1 is stated exclusively in terms of the location of its spectrum in the complex plane, and hence, is an intrinsically qualitative statement (cf. $[1,2,4])$.

From Theorem 4.1 and [4, Theorem 3.1], the latter characterizing the Roumieu-type strong Gevrey ultradifferentiability of all weak solutions of equation (1.2) of order $\beta \geq 1$ on $(0, \infty)$, we derive:

Corollary 4.1. Let $A$ be a scalar type spectral operator in a complex Banach space and $1 \leq \beta<\infty$. All weak solutions of equation (1.2) are $\beta$ th-order Roumie-type Gevrey ultradifferentiable on $(0, \infty)$ iff all weak solutions of equation (1.1) are Bth-order Beurling-type Gevrey ultradifferentiable on $\mathbb{R}$.

For $\beta=1$, we obtain the following important particular case.

Corollary 4.2. (Characterization of the entireness of weak solutions)

Let $A$ be a scalar type spectral operator in a complex Banach space. Every weak solution of equation (1.1) is an entire vector function iff there exist $b_{+}>0$ and $b_{-}>0$ such that the set $\sigma(A) \backslash \mathscr{P}_{b_{-}, b_{+}}^{1}$, where

$$
\mathscr{P}_{b, b_{+}}^{1}:=\left\{\lambda \in \mathbb{C}\left|\operatorname{Re} \lambda \leq-b_{-}\right| \operatorname{Im} \lambda \mid \quad \text { or } \operatorname{Re} \lambda \geq b_{+}|\operatorname{Im} \lambda|\right\},
$$

is bounded (see Figure 2).

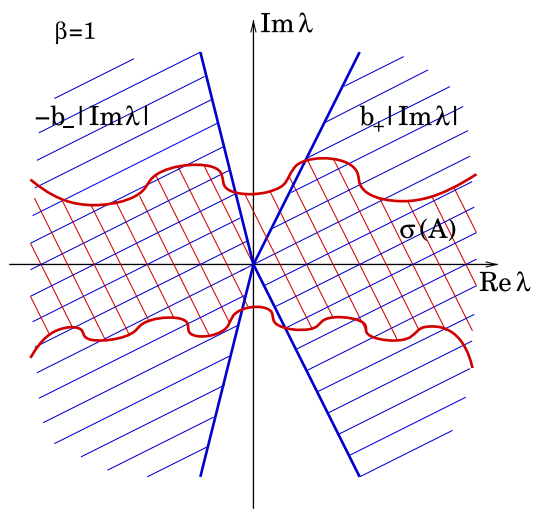

Figure 2: The case of $\beta=1$.

Remark 4.2. As is established in [1], all weak solutions of equation (1.1) with a scalar type spectral operator $A$ in a complex Banach space can be strongly infinite differentiable, while the operator $A$ is unbounded. Corollary 4.2 further implies that all weak solutions of equation (1.1) with a scalar type spectral operator $A$ in a complex Banach space can be entire vector functions without $A$ being bounded, e.g., when $A$ is an unbounded self-adjoint operator in a complex Hilbert space (cf. Corollary 7.3, [36, Corollary 4.1], and [36, Corollary 5.1]). This fact contrasts the situation when a closed densely defined linear operator $A$ in a complex Banach space generates a strongly continuous group $\{T(t)\}_{t \in \mathbb{R}}$ of bounded linear operators, i.e., the associated ACP is well-posed (see Remark 2.1), in which case even the (left or right) strong differentiability of all weak solutions of equation (2.1) at 0 immediately implies boundedness for $A$ (cf., e.g., [7]).

\section{Inherent smoothness improvement effects}

Theorem 4.1 implies, in particular, that

if, for some $1 \leq \beta<\infty$, every weak solution of equation (1.1) with a scalar type spectral operator A in a complex Banach space X belongs to the Bth-order Roumieu-type Gevrey class $\mathscr{E}^{\{\beta\}}(\mathbb{R}, X)$, then all of them belong to the narrower $\beta$ th-order Beurling-type Gevrey class $\mathscr{E}\{\beta\}(\mathbb{R}, X)$. 
Such a jump-like inherent smoothness improvement effect replicates the situation of the strong Gevrey ultradifferentiability of weak solutions of equation $(1.2)$ on $[0, \infty)[2$, Theorem 4.1].

Notably, for $\beta=1$, we have:

if every weak solution of equation (1.1) with a scalar type spectral operator A in a complex Banach space $X$ is analytically continuable into a complex neighborhood of $\mathbb{R}$ (each one into its own), then all of them are entire vector functions,

which can be further strengthened as follows.

Proposition 5.1. (Smoothness improvement effect)

Let $A$ be a scalar type spectral operator in a complex Banach space $(X,\|\cdot\|)$. If every weak solution of equation (1.1) is analytically continuable into a complex neighborhood of 0 (each one into its own), then all of them are entire vector functions.

Proof. Let us first show that if a weak solution $y(\cdot)$ of equation (1.1) is analytically continuable into a complex neighborhood of 0 , then $y(0)$ is an analytic vector of the operator $A$, i.e.,

$$
y(0) \in \mathscr{E}^{\{1\}}(A) .
$$

Let a weak solution $y(\cdot)$ of equation (1.1) be analytically continuable into a complex neighborhood of 0 . This implies that there is a $\delta>0$ such that

$$
y(t)=\sum_{n=0}^{\infty} \frac{y^{(n)}(0)}{n !} t^{n}, \quad t \in[-\delta, \delta] .
$$

The power series converging at $t=\delta$, there is a $c>0$ such that

$$
\left\|\frac{y^{(n)}(0)}{n !} \delta^{n}\right\| \leq c, \quad n \in Z_{+} .
$$

Whence, considering that, by Proposition 3.1 with $I=[-\delta, \delta]$,

$$
y(0) \in C^{\infty}(A) \quad \text { and } \quad y^{(n)}(0)=A^{n} y(0), \quad n \in \mathbb{Z}_{+},
$$

we infer that

$$
\left\|A^{n} y(0)\right\|=\left\|y^{(n)}(0)\right\| \leq c\left[\delta^{-1}\right]^{n} n !, \quad n \in \mathbb{Z}_{+},
$$

which implies

$$
y(0) \in \mathscr{E}^{\{1\}}(A) .
$$

Now, let us prove the statement by contrapositive assuming that there is a weak solution of equation (1.1), which is not an entire vector function. This, by Theorem 4.1 with $\beta=1$, implies that there is a weak solution $y(\cdot)$ of equation (1.1), which is not analytically continuable into a complex neighborhood of $\mathbb{R}$. Then, by Proposition 3.1, for some $t_{0} \in \mathbb{R}$,

$$
y\left(t_{0}\right) \notin \mathscr{E}^{\{1\}}(A)
$$

Therefore, for the weak solution

$$
y_{t_{0}}(t):=y\left(t+t_{0}\right), \quad t \in \mathbb{R},
$$

of equation (1.1),

$$
y_{t_{0}}(0)=y\left(t_{0}\right) \notin \mathscr{E}^{\{1\}}(A),
$$

which, as is shown above, implies that $y_{t_{0}}(\cdot)$ is not analytically continuable into a complex neighborhood of 0 , and hence, completes the proof by contrapositive.

Proposition 5.1 replicates the similar situation for weak solutions of equation (1.2) [2, Proposition 5.1]. 


\section{Gevrey ultradifferentiability of orders less than one}

While Corollary 4.2 implies that all weak solutions of equation (1.1) with a scalar type spectral operator $A$ in a complex Banach space can be entire vector functions without the operator's $A$ being bounded (see Remark 4.2), the following analogue of [3, Theorem 4.1] shows that $A$ cannot remain unbounded, if each weak solution $y(\cdot)$ of $(1.1)$, in addition to being entire, satisfies the growth estimate

$$
\|y(z)\| \leq M e^{\gamma|z|^{1 /(1-\beta)}}, \quad z \in \mathbb{C},
$$

with some $0 \leq \beta<1, \gamma>0$, and $M>0$ depending on $y(\cdot)$ (see (2.14)).

Theorem 6.1. (Gevrey ultradifferentiability of orders less than one)

Let $A$ be a scalar type spectral operator in a complex Banach space $(X,\|\cdot\|)$ with spectral measure $E_{A}(\cdot)$. If every weak solution of equation (1.1) belongs to the $\beta$ th-order Roumieu-type Gevrey class $\mathscr{E}^{\{\beta\}}(\mathbb{R}, X)$ with some $\beta \in[0,1)$ (each one to its own), then the operator $A$ is bounded, and hence, all weak solutions of equation (1.1) are necessarily entire vector functions of exponential type.

Proof. For the scalar type spectral operators

$$
A_{-}:=A E_{A}(\{\lambda \in \sigma(A) \mid \operatorname{Re} \lambda<0\})
$$

and

$$
A_{+}:=A E_{A}(\{\lambda \in \sigma(A) \mid \operatorname{Re} \lambda \geq 0\}),
$$

by the properties of the operational calculus (see [11, Theorem XVIII.2.11]),

$$
A=A_{-}+A_{+} .
$$

Let

$$
A_{1}:=A \mid E_{A}(\{\lambda \in \sigma(A) \mid \operatorname{Re} \lambda<0\}) X
$$

and

$$
A_{2}:=A \mid E_{A}(\{\lambda \in \sigma(A) \mid \operatorname{Re} \lambda \geq 0\}) X,
$$

where $\cdot$ is the restriction of an operator (left) to a subspace (right).

Since, by the properties of the operational calculus, the complementary subspaces

$$
E_{A}(\{\lambda \in \sigma(A) \mid \operatorname{Re} \lambda \geq 0\}) X \quad \text { and } \quad E_{A}(\{\lambda \in \sigma(A) \mid \operatorname{Re} \lambda \geq 0\}) X
$$

reduce the operators $A, A_{-}$, and $A_{+}$,

$$
\begin{aligned}
& \sigma(A)=\sigma\left(A_{1}\right) \cup \sigma\left(A_{2}\right), \\
& \sigma\left(A_{1}\right) \subseteq \sigma\left(A_{-}\right) \subseteq \sigma\left(A_{1}\right) \cup\{0\}, \\
& \sigma\left(A_{2}\right) \subseteq \sigma\left(A_{+}\right) \subseteq \sigma\left(A_{2}\right) \cup\{0\}
\end{aligned}
$$

(cf. [37]), with $\sigma\left(A_{i}\right)=\varnothing, i=1,2$, provided the underlying subspace is $\{0\}$.

Therefore, we have the following inclusions:

$$
\sigma(A) \subseteq \sigma\left(A_{-}\right) \cup \sigma\left(A_{+}\right) \subseteq \sigma(A) \cup\{0\} .
$$

By the properties of the operational calculus (see [11, Theorem XVIII.2.11]),

$$
\begin{aligned}
e^{t A_{+}} & =\int_{\sigma(A)} e^{t \lambda \lambda_{\{\lfloor\in \sigma(A) \mid \operatorname{Re} \lambda \geq 0\}}(\lambda)} \mathrm{d} E_{A}(\lambda)=\int_{\{\lambda \in \sigma(A) \mid \operatorname{Re} \lambda \geq 0\}} e^{t \lambda} \mathrm{d} E_{A}(\lambda)+\int_{\{\lambda \in \sigma(A) \mid \operatorname{Re} \lambda<0\}} 1 \mathrm{~d} E_{A}(\lambda) \\
& =e^{t A} E_{A}(\{\lambda \in \sigma(A) \mid \operatorname{Re} \lambda \geq 0\})+E_{A}(\{\lambda \in \sigma(A) \mid \operatorname{Re} \lambda<0\}), \quad t \in \mathbb{R},
\end{aligned}
$$

$\left(\chi_{\delta}(\cdot)\right.$ is the characteristic function of a set $\left.\delta \subseteq \mathbb{C}\right)$. 
Let

$$
f \in \bigcap_{t \geq 0} D\left(e^{t A_{+}}\right)
$$

be arbitrary. Then, by (6.42),

$$
E_{A}(\{\lambda \in \sigma(A) \mid \operatorname{Re} \lambda \geq 0\}) f \in \bigcap_{t \geq 0} D\left(e^{t A}\right) .
$$

Since, for arbitrary $t \in \mathbb{R}$ and any Borel set $\delta \subseteq \mathbb{C}$,

$$
\int_{\delta} e^{t \lambda} \mathrm{d} v\left(E_{A}(\{\lambda \in \sigma(A) \mid \operatorname{Re} \lambda \geq 0\}) f, g^{\star}, \lambda\right)=\int_{\delta \cap\{\lambda \in \sigma(A) \mid \operatorname{Re} \lambda \geq 0\}} e^{t \lambda} \mathrm{d} v\left(f, g^{\star}, \lambda\right)
$$

(see, e.g., [3, Preliminaries]), by Proposition 2.1, we infer that

$$
E_{A}(\{\lambda \in \sigma(A) \mid \operatorname{Re} \lambda \geq 0\}) f \in \bigcap_{t<0} D\left(e^{t A}\right) .
$$

Hence, for any $f \in \bigcap_{t \geq 0} D\left(e^{t A_{+}}\right)$,

$$
E_{A}(\{\lambda \in \sigma(A) \mid \operatorname{Re} \lambda \geq 0\}) f \in \bigcap_{t \in \mathbb{R}} D\left(e^{t A}\right) .
$$

This, by [21, Theorem 4.2], implies that every weak solution

$$
e^{t A_{+}} f=e^{t A} E_{A}(\{\lambda \in \sigma(A) \mid \operatorname{Re} \lambda \geq 0\}) f+E_{A}(\{\lambda \in \sigma(A) \mid \operatorname{Re} \lambda<0\}) f, \quad t \geq 0,
$$

where

$$
f \in \bigcap_{t \geq 0} D\left(e^{t A_{+}}\right)
$$

is arbitrary, of the equation

$$
y^{\prime}(t)=A_{+} y(t), \quad t \geq 0,
$$

along with the weak solution

$$
e^{t A} E_{A}(\{\lambda \in \sigma(A) \mid \operatorname{Re} \lambda \geq 0\}) f, \quad t \in \mathbb{R},
$$

of equation (1.1) and the constant vector function

$$
E_{A}(\{\lambda \in \sigma(A) \mid \operatorname{Re} \lambda<0\}) f, \quad t \in \mathbb{R},
$$

belongs to the $\beta$ th-order Roumieu-type Gevrey class $\mathscr{E}^{\{\beta\}}([0, \infty), X)$ with some $\beta \in[0,1)$ (each one to its own), which, by [3, Theorem 4.1], implies that the operator $A_{+}$is bounded, and hence, the spectrum $\sigma\left(A_{+}\right)$is a bounded set in $\mathbb{C}$ (see, e.g., $[18,38])$.

Using similar reasoning for the scalar type spectral operator $-A_{-}$and the evolution equation

$$
y^{\prime}(t)=-A_{-} y(t), \quad t \geq 0,
$$

(see Remarks 2.1), one can show that the spectrum of the operator $-A_{-}$, and hence, of the operator $A_{-}$is a bounded set in $\mathbb{C}$.

Thus, from inclusion (6.41), we infer that $\sigma(A)$ is a bounded set in $\mathbb{C}$, which, by [11, Theorem XVIII.2.11 (c)], means that the operator $A$ is bounded and completes the proof implying that every weak solution $y(\cdot)$ of equation (1.1) is an entire vector function of the form

$$
y(z)=e^{z A} f=\sum_{n=0}^{\infty} \frac{z^{n}}{n !} A^{n} f, \quad z \in \mathbb{C}, \text { with some } f \in X,
$$

and hence, satisfying the growth condition

$$
\|y(z)\| \leq\|f\| e^{\|A\||| z \mid}, \quad z \in \mathbb{C},
$$

is of exponential type (see Preliminaries). 


\section{The case of a normal operator}

As an important particular case of Theorem 4.1, we obtain:

Corollary 7.1. (Gevrey ultradifferentiability of order $\beta \geq 1$ )

Let $A$ be a normal operator in a complex Hilbert space $X$ and $1 \leq \beta<\infty$. Then the following statements are equivalent.

(i) Every weak solution of equation (1.1) belongs to the $\beta$ th-order Beurling-type Gevrey class $\mathscr{E}^{(\beta)}(\mathbb{R}, X)$.

(ii) Every weak solution of equation (1.1) belongs to the $\beta$ th-order Roumieu-type Gevrey class $\mathscr{E}^{\{\beta\}}(\mathbb{R}, X)$.

(iii) There exist $b_{+}>0$ and $b_{-}>0$ such that the set $\sigma(A) \backslash \mathscr{P}_{b_{-}, b_{+}}^{\beta}$, where

$$
\mathscr{P}_{b_{-}, b_{+}}^{\beta}:=\left\{\left.\lambda \in \mathbb{C}\left|\operatorname{Re} \lambda \leq-b_{-}\right| \operatorname{Im} \lambda\right|^{1 / \beta} \text { or } \operatorname{Re} \lambda \geq b_{+}|\operatorname{Im} \lambda|^{1 / \beta}\right\},
$$

is bounded (see Figure 1).

Remark 7.1. Corollary 7.1 develops the results of paper [36], where similar consideration is given to the strong Gevrey ultradifferentiability of the weak solutions of equation (1.2) with a normal operator in a complex Hilbert space on $[0, \infty)$ and $(0, \infty)$.

For $\beta=1$, we obtain the following important particular case.

Corollary 7.2. (Characterization of the entireness of weak solutions)

Let $A$ be a normal operator in a complex Hilbert space. Every weak solution of equation (1.1) is an entire vector function iff there exist $b_{+}>0$ and $b_{-}>0$ such that the set $\sigma(A) \backslash \mathscr{P}_{b_{-}, b_{+}}^{\beta}$, where

$$
\mathscr{P}_{b_{-}, b_{+}}^{\beta}:=\left\{\lambda \in \mathbb{C}\left|\operatorname{Re} \lambda \leq-b_{-}\right| \operatorname{Im} \lambda \mid \text { or } \operatorname{Re} \lambda \geq b_{+}|\operatorname{Im} \lambda|\right\},
$$

is bounded (see Figure 2).

Considering that, for a self-adjoint operator $A$ in a complex Hilbert space $X$,

$$
\sigma(A) \subseteq \mathbb{R}
$$

(see, e.g., [12,13]), by Corollary 7.2, we can strengthen [1, Corollary 18] as follows.

Corollary 7.3. (The case of a self-adjoint operator)

Every weak solution of equation (1.1) with a self-adjoint operator $A$ in a complex Hilbert space is an entire vector function.

Cf. [36, Corollary 4.1] and [36, Corollary 5.1].

From Corollary 4.1, we immediately obtain:

Corollary 7.4. Let $A$ be a normal operator in a complex Hilbert space and $1 \leq \beta<\infty$. All weak solutions of equation (1.2) are $\beta$ th-order Roumie-type Gevrey ultradifferentiable on $(0, \infty)$ iff all weak solutions of equation (1.1) are $\beta$ th-order Beurling-type Gevrey ultradifferentiable on $\mathbb{R}$.

Cf. [36, Theorem 4.2].

For a normal operator in a complex Hilbert space, Proposition 5.1 and Theorem 6.1 acquire the following form, respectively.

Corollary 7.5. (Smoothness improvement effect)

Let $A$ be a normal operator in a complex Hilbert space. If every weak solution of equation (1.1) is analytically continuable into a complex neighborhood of $O$ (each one into its own), then all of them are entire vector functions. 
Corollary 7.6. (Gevrey ultradifferentiability of orders less than one)

Let $A$ be a normal operator in a complex Hilbert space X. If every weak solution of equation (1.1) belongs to the $\beta$ th-order Roumieu type Gevrey class $\mathscr{E}^{\mathfrak{S}\}}(\mathbb{R}, X)$ with $0 \leq \beta<1$ (each one to its own), then the operator $A$ is bounded, and hence, all weak solutions of equation (1.1) are necessarily entire vector functions of exponential type.

Observe that Corollary 7.5 replicates the similar situation for weak solutions of equation (1.2) with a normal operator in a complex Hilbert space [36, Proposition 5.1].

Acknowledgment: The author's appreciation goes to his colleague, Dr. Maria Nogin of the Department of Mathematics, California State University, Fresno, for her kind assistance with the graphics.

\section{References}

[1] M. V. Markin, On the differentiability of weak solutions of an abstract evolution equation with a scalar type spectral operator on the real axis, Int. J. Math. Math. Sci. 2018 (2018), 4168609.

[2] M. V. Markin, On the Gevrey ultradifferentiability of weak solutions of an abstract evolution equation with a scalar type spectral operator, Methods Funct. Anal. Topology 24 (2018), no. 4, 349-369.

[3] M. V. Markin, On the Gevrey ultradifferentiability of weak solutions of an abstract evolution equation with a scalar type spectral operator of orders less than one, Open Math. 17 (2019), no. 1, 1-14.

[4] M. V. Markin, On the Gevrey ultradifferentiability of weak solutions of an abstract evolution equation with a scalar type spectral operator on the open semi-axis, Open Math. 17 (2019), no. 1, 1082-1112.

[5] M. V. Markin, On the mean ergodicity of weak solutions of an abstract evolution equation, Methods Funct. Anal. Topology 24 (2018), no. 1, 53-70.

[6] J. M. Ball, Strongly continuous semigroups, weak solutions, and the variation of constants formula, Proc. Amer. Math. Soc. 63 (1977), no. 2, 370-373.

[7] K.-J. Engel and R. Nagel, One-Parameter Semigroups for Linear Evolution Equations, Graduate Texts in Mathematics, vol. 194, Springer-Verlag, New York, 2000.

[8] E. Hille and R. S. Phillips, Functional Analysis and Semi-groups, American Mathematical Society Colloquium Publications, vol. 31, Amer. Math. Soc., Providence, RI, 1957.

[9] N. Dunford, Spectral operators, Pacific J. Math. 4 (1954), 321-354.

[10] N. Dunford, A survey of the theory of spectral operators, Bull. Amer. Math. Soc. 64 (1958), 217-274.

[11] N. Dunford and J. T. Schwartz with the assistance of W. G. Bade and R. G. Bartle, Linear Operators. Part III: Spectral Operators, Interscience Publishers, New York, 1971.

[12] N. Dunford and J. T. Schwartz with the assistance of W. G. Bade and R. G. Bartle, Linear Operators. Part II: Spectral Theory. Self Adjoint Operators in Hilbert Space, Interscience Publishers, New York, 1963.

[13] A. I. Plesner, Spectral Theory of Linear Operators, Nauka, Moscow, 1965 (Russian).

[14] J. Wermer, Commuting spectral measures on Hilbert space, Pacific J. Math. 4 (1954), 355-361.

[15] M. V. Markin, A note on the spectral operators of scalar type and semigroups of bounded linear operators, Int. J. Math. Math. Sci. 32 (2002), no. 10, 635-640.

[16] E. Berkson, Semi-groups of scalar type operators and a theorem of Stone, Illinois J. Math. 10 (1966), no. 2, 345-352.

[17] T. V. Panchapagesan, Semi-groups of scalar type operators in Banach spaces, Pacific J. Math. 30 (1969), no. 2, $489-517$.

[18] N. Dunford and J. T. Schwartz with the assistance of W. G. Bade and R. G. Bartle, Linear Operators. Part I: General Theory, Interscience Publishers, New York, 1958.

[19] M. V. Markin, On scalar type spectral operators, infinite differentiable and Gevrey ultradifferentiable $C_{O^{-}}$-semigroups, Int. J. Math. Math. Sci. 2004 (2004), no. 45, 2401-2422.

[20] M. V. Markin, On the Carleman classes of vectors of a scalar type spectral operator, Int. J. Math. Math. Sci. 2004 (2004), no. $60,3219-3235$.

[21] M. V. Markin, On an abstract evolution equation with a spectral operator of scalar type, Int. J. Math. Math. Sci. 32 (2002), no. $9,555-563$.

[22] M. Gevrey, Sur la nature analytique des solutions des équations aux dérivées partielles, Ann. Éc. Norm. Sup. Paris 35 (1918), 129-196.

[23] H. Komatsu, Ultradistributions and Hyperfunctions. Hyperfunctions and Pseudo-Differential Equations, Lecture Notes in Math., vol. 287, Springer, Berlin, 1973, pp. 164-179. 
[24] H. Komatsu, Ultradistributions. I. Structure theorems and a characterization, J. Fac. Sci. Univ. Tokyo Sect. IA Math. 20 (1973), 25-105.

[25] H. Komatsu, Microlocal Analysis in Gevrey Classes and in Complex Domains, Lecture Notes in Math., vol. 1495, Springer, Berlin, 1991, pp. 161-236.

[26] S. Mandelbrojt, Séries de Fourier et Classes Quasi-Analytiques de Fonctions, Gauthier-Villars, Paris, 1935.

[27] M. V. Markin, On the strong smoothness of weak solutions of an abstract evolution equation. III. Gevrey ultradifferentiability of orders less than one, Appl. Anal. 78 (2001), no. 1-2, 139-152.

[28] B. Ja. Levin, Distribution of Zeros of Entire Functions, Translations of Mathematical Monographs, vol. 5, American Mathematical Society, Providence, RI, 1980.

[29] V. I. Gorbachuk, Spaces of infinitely differentiable vectors of a nonnegative self-adjoint operator, Ukrainian Math. J. 35 (1983), 531-534.

[30] V. I. Gorbachuk and M. L. Gorbachuk, Boundary Value Problems for Operator Differential Equations, Mathematics and Its Applications (Soviet Series), vol. 48, Kluwer Academic Publishers Group, Dordrecht, 1991.

[31] V. I. Gorbachuk and A. V. Knyazyuk, Boundary values of solutions of operator-differential equations, Russ. Math. Surveys 44 (1989), 67-111.

[32] E. Nelson, Analytic vectors, Ann. of Math. 70 (1959), no. 3, 572-615.

[33] R. Goodman, Analytic and entire vectors for representations of Lie groups, Trans. Amer. Math. Soc. 143 (1969), 55-76.

[34] Ya. V. Radyno, The space of vectors of exponential type, Dokl. Akad. Nauk BSSR 27 (1983), no. 9, 791-793 (Russian with English summary).

[35] M. V. Markin, On the Carleman ultradifferentiable vectors of a scalar type spectral operator, Methods Funct. Anal. Topology 21 (2015), no. 4, 361-369.

[36] M. V. Markin, On the strong smoothness of weak solutions of an abstract evolution equation. II. Gevrey ultradifferentiability, Appl. Anal. 78 (2001), no. 1-2, 97-137.

[37] M. V. Markin, On certain spectral features inherent to scalar type spectral operators, Methods Funct. Anal. Topology 23 (2017), no. 1, 60-65.

[38] M. V. Markin, Elementary Operator Theory, De Gruyter Graduate, Walter de Gruyter GmbH, Berlin/Boston, 2020. 